\title{
Stationary and Transition Probabilities in Slow Mixing, Long Memory Markov Processes
}

\author{
Meysam Asadi, Student Member, IEEE, Ramezan Paravi Torghabeh, Student Member, IEEE, \\ and Narayana P. Santhanam, Member, IEEE,
}

\begin{abstract}
We observe a length- $n$ sample generated by an unknown, stationary ergodic Markov process (model) over a finite alphabet $\mathcal{A}$. Given any string $\mathrm{w}$ of symbols from $\mathcal{A}$ we want estimates of the conditional probability distribution of symbols following $\mathrm{w}$, as well as the stationary probability of $\mathrm{w}$. Two distinct problems that complicate estimation in this setting are (i) long memory, and (ii) slow mixing which could happen even with only one bit of memory.

Any consistent estimator in this setting can only converge pointwise over the class of all ergodic Markov models. Namely, given any estimator and any sample size $n$, the underlying model could be such that the estimator performs poorly on a sample of size $n$ with high probability. But can we look at a length- $n$ sample and identify if an estimate is likely to be accurate?

Since the memory is unknown a-priori, a natural approach is to estimate a potentially coarser model with memory $k_{n}=\mathcal{O}(\log n)$. As $n$ grows, pointwise consistent estimates that hold eventually almost surely (e.a.s.) are known so long as the scaling of $k_{n}$ is not superlogarithmic in $n$. Here, rather than e.a.s. convergence results, we want the best answers possible with a length- $n$ sample. Combining results in universal compression with Aldous' coupling arguments, we obtain sufficient conditions on the length$n$ sample (even for slow mixing models) to identify when naive (i) estimates of the conditional probabilities and (ii) estimates related to the stationary probabilities are accurate; and also bound the deviations of the naive estimates from true values.
\end{abstract}

Index Terms-Context-tree weighting, Coupling, Markov processes, Pointwise consistency, Universal compression.

\section{INTRODUCTION}

$\mathbf{W}$ E explore the question of estimating a stationary ergodic $\mathcal{A}$-ary Markov process (model) from a length- $n$ sample generated by it. Ideally, given any string $\mathbf{w}$ of symbols from $\mathcal{A}$ we want an estimate of the conditional probability distribution of symbols following $\mathbf{w}$, as well as the stationary probability of $\mathbf{w}$. As with PAC-learning setup [1], estimates should come with an accuracy guarantee which holds with a certain confidence.

For the simpler, finite alphabet i.i.d. sources, empirical probabilities estimated from length- $n$ samples are well understood. The deviation of empirical estimates from true values is characterized by the Chernoff bound [2] and generalized

The authors are with the Department of Electrical Engineering, University of Hawai'i, Mānoa, Honolulu, HI 96822 USA (Email:\{masadi, paravi, nsanthan\}@ hawaii.edu).

Manuscript received May 3, 2013; revised January 14, 2014; accepted April 19, 2014.

This paper was presented in part at the 2013 IEEE International Symposium on Information Theory.

Copyright (c) 2013 IEEE. Personal use of this material is permitted. However, permission to use this material for any other purposes must be obtained from the IEEE by sending a request to pubs-permissions @ ieee.org. by Hoeffding bounds [3]. On the other hand, Markov sources contain additional biases which have to do with the mixing of the source, or how quickly all states of the process are explored.

Roughly speaking, an aperiodic, ergodic source has mixed (or explored all states properly) when the empirical counts of states in the sample reflects their stationary probabilities. Given a source has mixed, it is therefore possible to estimate the transition probabilities using the stationary probabilities. Indeed, most estimation (theoretical or in practice) follows this sequence of logic. Most theoretical results prove that the empirical counts of states reflect their stationary probabilities eventually almost surely, and build on this to obtain transition probabilities.

In this paper, we are interested in the regime when the source has not yet mixed. This breaks the usual approachwhen the empirical counts of states do not reflect stationary probabilities, is it at all possible to estimate the transition probabilities? And when the counts of states are not near their stationary probabilities, what do they actually signify?

\section{Motivation: Information aggregation on the Internet}

In this paper we focus on the theoretical underpinnings of Markov estimation in slow mixing regime. However, it may be instructive to consider the following problem that places estimation in the slow mixing setting in a concrete context.

There is implicit but not well-modeled bias in widely adopted means of obtaining news and other information on the Internet. Rather than one news channel disseminating information over TV or radio as in times not too long ago, there is a very broad choice today among news sources. While it is desirable that citizens are exposed to a variety of information sources that is not what really happens in, say, a political context. Depending on political persuasion, one starts off with perhaps the conservative Fox News web portal or maybe the liberal New York Times. These sites would then link to various blogs, more news web sites, and so on-but perhaps mostly on the same side of the political spectrum. Even in webpages that do not clearly fall into either side, probabilities with which links are chosen are reflective of the user opinions. Common browsing habits are therefore not likely to explore the diversity of views on the Internet, but rather be confined to sections of the network and perhaps consider certain opinions or news more than others. Of course, not all news is polarized-sports scores, for example, are not.

Contrast the above with a browsing model that motivates a different view of the Internet—-the Google PageRank. Here the 
hypothetical user follows links at random from the page she is currently on. In addition, the user may jump to a random page on the Internet with a specified (reset) probability. Such a random walk is fast-mixing [4], rendering its allied global properties like PageRank easy to compute. While PageRank's efficacy in search tasks is quite self-evident, it does not capture opinions of specific users. Both the NYT and Fox News may have high PageRank but very few in the United States would rate/use both these portals highly.

Any walk on a graph with a randomized aspect to it naturally defines a Markov process made of symbols corresponding to the vertices of the graph. To study the polarization of views on a topic, suppose we represent each page by a finite-alphabet signature relevant to the topic at hand. For example, at the very simplest, consider representing each page by a bit that represents the presence or absence of a particular combination of keywords. Secondly, we make the modeling assumption that browsing history captures users preferences-namely, if we were given the sum total of a user's browsing histories, we could obtain the probabilities with which the user may follow various pages.

We therefore model the problem with a Markov process $p$ defined by a full binary tree whose leaves are represented by a suffix-free set $\mathcal{T} \subset\{0,1\}^{*}$. The leaves of $\mathcal{T}$ are the states (browsing histories) of the process and $Y_{i}$ is the $i^{\prime}$ th page visited. Let $\mathbf{c}_{\mathcal{T}}\left(Y_{-\infty}^{0}\right)$ be the longest suffix of $Y_{-\infty}^{0}$ in $\mathcal{T}$, then

$$
p\left(Y_{1}^{n} \mid Y_{-\infty}^{0}\right)=\prod_{i=1}^{n} p\left(Y_{i} \mid \mathbf{c}_{\mathcal{T}}\left(Y_{-\infty}^{i-1}\right)\right) .
$$

All users adhere to this general unknown model $p$, but users are distinguished by modeling their distinct opinions/preferences as the transition probabilities corresponding to different states (browsing histories) of the process above. It can be shown (e.g.,, via the Dobrushin coefficient) that the more polarized user opinions are, the slower mixing $p$ is. Note that here, it is reasonable to assume that the incremental information an additional page in the browser history provides diminishes with the amount of history we already have.

Given a topic, how would one describe the polarization of opinion or information of that topic? In our formalization, quantifying polarization amounts to obtaining the transition and stationary probabilities of the process $p$ above. To elucidate, we ask two questions.

What user profiles can we tell apart? We do not know $\mathcal{T}$ the browsing contexts that fix user preferences and click probabilities. So, with a finite amount of browsing data, the best we can ask for is to estimate the click probabilities $p_{\mathcal{T}, q}\left(Y_{1} \mid \mathbf{w}\right)$ for contexts $\mathbf{w}$ where $\mathbf{w} \in\{0,1\}^{k_{n}}$ for some number $k_{n}$ that depends on the amount $n$ of browsing data we have. As we will see, in the slow mixing case, not all contexts that appear in the browsing data may be amenable to modeling these probabilities.

What is the global picture? The stationary probabilities of $\mathbf{w}$ that appear in the browsing data, reflecting how prevalant the distinguishable user profiles are (how relevant each one is).

If we were to translate our theoretical approach in this paper to a one line layman summary of how to quantify polarization, we would ask how much common information should different users see before they begin to agree. While we have described the motivation that lies behind the slow mixing formulation, this paper focuses on the general theoretical and statistical underpinnings of the two problems above (transition and stationary probabilities of slow mixing Markov processes) rather than the details of how to aggregate information.

\section{Outline of problem}

We first consider two complications while estimating Markov processes in the slow mixing setting.

Difficulties: The first complication is that irrespective of how large the size of the sample at hand, $n$, is we may not be in a position to reliably provide estimates of the stationary probabilities.

Consider a length- $n$ sample obtained from the following binary Markov source with memory one. The transition probability from 1 to 0 in a memory- 1 source is $\epsilon \ll 1 / n$. By changing the transition probability from 0 to 1 appropriately, we can vary the stationary probabilities of $1 \mathrm{~s}$ and $0 \mathrm{~s}$ in a wide range without changing how a length- $n$ sample will look like. As specific examples, consider two binary, one-bit memory Markov sources; the first assigns the transition probability from 0 to 1 to be $\epsilon$, while the second assigns $2 \epsilon$. An easy computation (see also Example 4 in Section IV) shows that the stationary probabilities of 1 and 0 are $(1 / 2,1 / 2)$ and $(2 / 3,1 / 3)$ respectively.

But, if we start from the context 1 , with high probability both sources will yield a sequence of $n 1 \mathrm{~s}$. We cannot distinguish between the two sources above with a sample of this size, and therefore it is futile to estimate stationary probabilities from this sample. This particular regime where the number of times each state ( 1 and 0 in this example) appears do not reflect their stationary probabilities is often formalized as the slow mixing case, see [5].

The second complication is that no matter what the sample size $n$ is, with high probability the set of all strings $\mathbf{w}$ (of any fixed length) in a length- $n$ sample may have arbitrarily small mass under the stationary distribution.

To see this, observe that a memory- 1 binary source that transitions from 1 to 0 with probability $1-\epsilon$ and 0 to 1 with probability $\epsilon / m$ has the stationary probability of 1 to be $1 /(m+1)$ (see Example 2 in Section IV). Yet if $\epsilon \ll 1 / n$, starting from state 1 we see a sequence of $n 1$ s with high probability. By making $m$ large enough, the probability of 1 (and therefore of any sequence of 1s) can be made arbitrarily small, illustrating the conundrum.

Problem: If the source cannot always be well estimated as above, we would like to give the best possible answer from the length- $n$ sample - one which may also depend on how the data looks. Say, for the sake of a concrete example, that we have a sample $\mathbf{x}_{1}$, with $n-\log n 1 \mathrm{~s}$ followed by a string of $\log n$ 0s. Perhaps, this may have come from a one-bit memory, slow mixing Markov source as in Example 4 As we saw, it is futile to estimate stationary probabilities in this case. Contrast this sample with a new sample $\mathbf{x}_{2}$, also with $n-\log n 1 \mathrm{~s}$ and $\log n 0$ s, but $\mathbf{x}_{2}$ has 0 s spread uniformly in the sequence. 
Unlike with $\mathbf{x}_{1}$, upon seeing $\mathbf{x}_{2}$ we may want to conclude that we have an i.i.d. source with a high probability for 1 .

We therefore ask how best to estimate properties of an ergodic, yet potentially slow mixing Markov process from a sample of size $n$. As the above example shows, we have an estimation problem where any estimator can only converge pointwise to the true values, rather than uniformly over the model class. Rather than e.a.s. guarantees, given a realization of a Markov process we attempt to provide deviation bounds for transition and stationary probabilities of substrings seen in the sample. We insist that our bounds, while being model dependent as is to be expected, must however be calculated using only parameters which are well-approximated from the data at hand.

If the Markov source is completely arbitrary, such bounds will essentially be trivial. Therefore, we make an assumption justified by the motivation we consider-that the information provided by a symbol $i$ positions in the past given everything in between diminishes with $i$. However, we do not assume apriori knowledge on the depth of context tree of the process, nor do we assume that the conditional probabilities given the pasts are bounded uniformly away from zero.

\section{Results}

We provide a short background on Markov processes in Section III. while Section $\mathrm{V}$ contains a formal summary of results.

At a high level, these results show how to look at a data sample and identify properties of the process that are amenable to accurate estimation from the sample even if the source is slow mixing. They also allow us to sometimes (depending on how the data looks) conclude that certain naive estimators of transition probabilities (Section X, Theorem 2), or stationary probabilities (Section XII] Theorem 7) happen to be accurate even if the process is slow mixing.

Contrary to most prior work, we first obtain estimates on transition probabilities. To do so, we use universal compression approaches that do not require that empirical counts of states be close to their stationary probability. Interpreting the empirical counts of states from the approximate transition probabilities is complicated by the fact that stationary probabilities can be very sensitive functions of the transition probabilities. What then can we say about empirical counts from the few approximate transition probabilities obtained from the sample? We use a coupling argument [6] in Section XII] to answer this question.

Finally, since our results do not rely on empirical counts of strings reaching their stationary probabilities, they could be strengthened using other arguments in literature in cases where we know that the counts do reflect stationary probabilities.

\section{Estimation and compression}

In the set of all Markov sources, mixing properties only affect estimation, and is irrelevant to universal compression [7], [8]. We have already seen how slow mixing rendered estimation of stationary probabilities impossible in general if we are only allowed a fixed length sample, no matter how large the sample size is. But the sequences on which estimation was impossible lend themselves to good universal compression.

This must give us a little pause since in the finite alphabet i.i.d. case, universal compression and estimation go hand in hand. Specifically, we will compare two cases-(i) the set of all i.i.d. binary models, and (ii) the set of all binary Markov models with memory one. The first i.i.d. collection is well compressible and universal compression algorithms with only sublinear redundancy $\Theta(\log n)$ exis 1 . Here, it is also possible to estimate the underlying distribution using a good universal compressor. Specific examples include the Krichevsky-Trofimov [9] (also known as the add 1/2 rule) or Laplace [10], [11] (add 1 rule) approaches. As a more complex example, the Good-Turing estimator (see [12]) can also be interpreted as being obtained from such a universal description [13] in a more general setting-where data is exchangeable, rather than i.i.d..

In the second Markov case as well, universal compression algorithms [14] can compress the data well, again with redundancy that is only sublinear as $\Theta(\log n)$. But universal compression algorithms cannot be used to always infer stationary properties of the source, as illustrated in the examples above. Put another way, while we may not be able to always estimate stationary properties of sources, we can compress sequences generated by slow mixing sources well.

\section{PRIOR WORK}

\section{A. Prior work on compression of Markov processes}

For Markov processes with known memory $k$, optimal redundancy rates for the universal compression and estimation have been established, see e.g., [15] for an overview and also [16], [17], [18]. These universal compression results imply consistent estimators for probabilities of sequences. Moreover, the rate of convergence of these estimators can be bounded uniformly over the entire memory $-k$ Markov model class e.g., [19], [20], [14], [21]. This rate typically depends exponentially on $k$ and diminishes with the sample length as $\log n / n$. We point out two complications when confronted with our problem.

First, we deal with the case of unbounded memory-namely no $a-$ prior $i$ bound on $k$. For the set of all finite memory Markov sources, only weakly universal [22] compression schemes - those that convergence in a pointwise sense-can be built (see [23] for a particularly nice construction). Namely, the convergence of the weak universal algorithms varies depending on the true unseen memory of the source. However, as we will see in Example 3, it may be impossible to estimate the memory of the source from a finite length sample. There has been a lot of work on the topic of estimating the memory of the source consistently when a prior bound on the memory is unknown, see [24], [25], [26], [27]—but as one would expect, given a finite length sample no estimator developed will always have a good answer.

Second, despite the positive result for estimation of the probabilities of sequences, as mentioned in the introduction

${ }^{1}$ A function $f_{n}=\Theta\left(g_{n}\right)$ if $f_{n}=\mathcal{O}\left(g_{n}\right)$ and $g_{n}=\mathcal{O}\left(f_{n}\right)$. 
there can not be estimators for transition and stationary probabilities whose rate of convergence is uniform over the entire model class. This negative observation follows simply because of the way we are forced to sample from slow mixing processes-and this is a complication compression does not encounter. For instance, in the example outlined above in the introduction, both samples $\mathbf{x}_{1}$ and $\mathbf{x}_{2}$ can be well compressed by universal estimators, but estimation is a whole different ballgame.

The complications above apart, the nature of questions we ask is different as well. Rather than consistency results, or establishing process-dependent rates of convergence for the case where the memory can be unbounded, we ask how to give the best possible answer with a given sample of length $n$. It is not to say, however, that the above compression results are irrelevant to our problem. Far from it, one of our results, Theorem 2 in Section X builds on (among other things) the universal compression results obtained for $k$-memory processes.

\section{B. Prior work on estimation of Markov processes}

Estimation for Markov processes has been extensively studied and falls into three major categories (i) consistency of estimators e.g., [28], [29], [24], [25], [30], (ii) guarantees on estimates that hold eventually almost surely e.g., [31], [32], and (iii) guarantees that hold for all sample sizes but which depend on both transition and stationary probabilities e.g., [26], [27], [33], [34]. The list above is not exhaustive, rather it focuses on the work closest to the approaches we take.

As mentioned earlier, performance of any estimator cannot not be bounded uniformly over all Markov models, something reflected in the line (iii) of research and in our work. While unavoidable, it poses a problem since the deviation bounds now depend on the unknown model. How then do we say if our estimate is doing well? Our thrust in this paper focuses on exactly this question-it is not just about consistency, rather that we want to gauge from the observed sample if our estimator is doing well relative to the unknown probability law in force.

In [30] a survey on consistent estimators for conditional probabilities of Markov processes is provided. For instance, given a realization of a Markov process, they provide a sequence of estimators for transition probabilities along some of time steps which converges almost surely to the true values.

Consistent estimators for the order of Markov processes have been studied in prior literature e.g., [35], [32], [36], [37]. In [32], [36], the penalized maximum likelihood technique is used to provide a consistent order estimator. In [37], a different consistent order estimator based on empirical counts is proposed which minimizes the asymptotic underestimation exponent while keeping the overestimation exponent at a certain level. Using the same technique, in [38] an optimal order estimator is provided, however they assume a prior upper bound on the memory of underlying process.

In [24], estimation of the minimal context tree of the Markov process is addressed. Two different information criteria, namely Bayesian Information Criterion and Minimum
Description Length are used, and consistency of estimation of the underlying context tree was established provided that the depth of hypothetical trees grow as $o(\log n) 2^{2}$ Moreover, it was shown in [25] that when the process has finite memory, the $o(\log n)$ condition is not necessary for estimation consistency.

In [26], [27], [34], exponential upper bounds on probability of incorrect estimation of (i) conditional and stationary probabilities and (ii) the underlying context tree, are provided for variants of Rissanen's algorithm context and penalized maximum likelihood estimator. The introduced deviation bounds depend on the model parameters (e.g., minimum stationary probability of all contexts $p_{\min }$, depth of the tree and continuity rate coefficients) of underlying process.

One particular paper that we would like to highlight is [33], where the problem of estimating a stationary ergodic process by finite memory Markov processes based on an $n$-length sample of the process is addressed. A measure of distance between the true process and its estimation is introduced and a convergence rate with respect to that measure is provided. However, the bounds proved there hold only when the infimum of conditional probabilities of symbols given the pasts are bounded away from zero.

In this paper, we see entropy estimation as a means of motivating the main problems to be posed. The best known results in the extensive research on entropy rate estimation for Markov processes are again related to Lempel-Ziv universal lossless data compression methods [39]. See [30] for a survey of other work as well.

\section{MARKOV PROCESSES}

Most notation, while standard, is included for completeness.

\section{A. Alphabet and strings}

$\mathcal{A}$ is a finite alphabet with cardinality $|\mathcal{A}|, \mathcal{A}^{*}=\bigcup_{k \geq 0} \mathcal{A}^{k}$ and $\mathcal{A}^{\infty}$ denotes the set of all semi-infinite strings of symbols in $\mathcal{A}$.

We denote the length of a string $\mathbf{u}=u_{1}, \ldots, u_{l} \in \mathcal{A}^{l}$ by $|\mathbf{u}|$, and use $\mathbf{u}_{i}^{j}=\left(u_{i}, \cdots, u_{j}\right)$. The concatenation of strings $\mathbf{w}$ and $\mathbf{v}$ is denoted by $\mathbf{w} \mathbf{v}$. A string $\mathbf{v}$ is a suffix of $\mathbf{u}$, denoted by $\mathbf{v} \preceq \mathbf{u}$, if there exists a string $\mathbf{w}$ such that $\mathbf{u}=\mathbf{w} \mathbf{v}$. A set $\mathcal{T}$ of strings is suffix-free if no string of $\mathcal{T}$ is a suffix of any other string in $\mathcal{T}$.

\section{B. Trees}

As in [14] for example, we use full $\mathcal{A}$-ary trees to represent the states of a Markov process. We denote full trees $\mathcal{T}$ as a suffix-free set $\mathcal{T} \subset \mathcal{A}^{*}$ of strings (the leaves) whose lengths satisfy Kraft's lemma with equality. The depth of the tree $\mathcal{T}$ is defined as $\kappa(\mathcal{T})=\max \{|\mathbf{u}|: \mathbf{u} \in \mathcal{T}\}$. A string $\mathbf{v} \in \mathcal{A}^{*}$ is an internal node of $\mathcal{T}$ if either $\mathbf{v} \in \mathcal{T}$ or there exists $\mathbf{u} \in \mathcal{T}$ such that $\mathbf{v} \preceq \mathbf{u}$. The children of an internal node $\mathbf{v}$ in $\mathcal{T}$, are those strings (if any) $a \mathbf{v}, a \in \mathcal{A}$ which are themselves either internal nodes or leaves in $\mathcal{T}$.

For any internal node $\mathbf{w}$ of a tree $\mathcal{T}$, let $\mathcal{T}_{\mathbf{w}}=\{\mathbf{u} \in \mathcal{T}$ : $\mathbf{w} \preceq \mathbf{u}\}$ be the subtree rooted at $\mathbf{w}$. Given two trees $\mathcal{T}_{1}$ and $\mathcal{T}_{2}$, we say that $\mathcal{T}_{1}$ is included in $\mathcal{T}_{2}\left(\mathcal{T}_{1} \preceq \mathcal{T}_{2}\right)$, if all the leaves in $\mathcal{T}_{1}$ are either leaves or internal nodes of $\mathcal{T}_{2}$.

\footnotetext{
${ }^{2}$ A function $f_{n}=o\left(g_{n}\right)$ if $\lim _{n \rightarrow \infty} f_{n} / g_{n}=0$.
} 


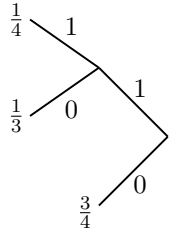

(a)

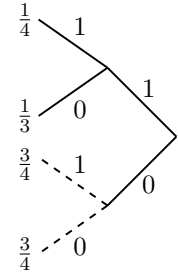

(b)
Fig. 1. (a) States and parameters of a binary Markov process in Example 1 (b) Same Markov process reparameterized to be a complete tree of depth 2 . We can similarly reparameterize the process on the left with a complete tree of any depth larger than 2 .

\section{Models}

Let $\mathcal{P}^{+}(\mathcal{A})$ be the set of all probability distributions on $\mathcal{A}$ such that every probability is strictly positive.

Definition 1. A context tree model is a finite full tree $\mathcal{T} \subset \mathcal{A}^{*}$ with a collection of probability distributions $q(\cdot \mid \mathbf{s}) \in$ $\mathcal{P}^{+}(\mathcal{A})$ assigned to each $\mathbf{s} \in \mathcal{T}$. We will refer to the elements of $\mathcal{T}$ as states (or contexts), and $q(\mathcal{T})=\{q(a \mid \mathbf{s}): \mathbf{s} \in \mathcal{T}, a \in$ $\mathcal{A}\}$ as the set of state transition probabilities.

Every model $(\mathcal{T}, q(\mathcal{T}))$ allows for an irreducible, aperiodic ${ }^{3}$ and ergodic [40] Markov process with a unique stationary distribution $\pi$ satisfying

$$
\pi Q=\pi,
$$

where $Q$ is the standard transition probability matrix formed using $q(\mathcal{T})$. Let $p_{\mathcal{T}, q}$ be the unique stationary Markov process $\left\{\ldots, Y_{0}, Y_{1}, Y_{2}, \ldots\right\}$ which takes values in $\mathcal{A}$ satisfying

$$
p_{\mathcal{T}, q}\left(Y_{1} \mid Y_{-\infty}^{0}\right)=q\left(Y_{1} \mid \mathbf{s}\right),
$$

where $\mathbf{s}$ is the unique suffix $\mathbf{s} \preceq Y_{-\infty}^{0}$ in $\mathcal{T}$ denoted by $\mathbf{c}_{\mathcal{T}}\left(Y_{-\infty}^{0}\right)$. Namely the mapping $\mathbf{c}_{\mathcal{T}}$ maps any (long enough) sequence to its unique suffix in $\mathcal{T}$. When the argument of $\mathbf{c}_{\mathcal{T}}$ is an internal node of $\mathcal{T}$, we leave the image of the mapping undefined.

As a note, when we write out actual strings in transition probabilities as in $q(0 \mid 1000)$, the state 1000 is the sequence of bits as we encounter them when reading the string left to right. If 0 follows $\cdots 1100$, the next state is a suffix of $\cdots 11000$, and if 1 follows $\cdots 1100$, the next state is a suffix of $\cdots 11001$.

Observation 1. A useful observation is that any model $(\mathcal{T}, q(\mathcal{T}))$ yields the same Markov process as a model $\left(\mathcal{T}^{\prime}, q\left(\mathcal{T}^{\prime}\right)\right)$ where $\mathcal{T} \preceq \mathcal{T}^{\prime}$ and for all $\mathbf{s}^{\prime} \in \mathcal{T}^{\prime}, q\left(\cdot \mid \mathbf{s}^{\prime}\right)=$ $q\left(\cdot \mid \mathbf{c}_{\mathcal{T}}\left(\mathbf{s}^{\prime}\right)\right)$.

Example 1. Let $(\mathcal{T}, q(\mathcal{T}))$ be a binary Markov process with $\mathcal{T}=\{11,01,0\}$ and $q(1 \mid 11)=\frac{1}{4}, q(1 \mid 01)=\frac{1}{3}, q(1 \mid 0)=$ $\frac{3}{4}$ as shown in Fig. 1. (a). Observe that Fig. 1. (b) shows the same Markov process as a model $\left(\mathcal{T}^{\prime}, q\left(\mathcal{T}^{\prime}\right)\right)$ with $\mathcal{T}^{\prime}=$ $\{11,01,10,00\}$ satisfying conditions in Observation 1 .

\footnotetext{
${ }^{3}$ Irreducible since $q(\cdot \mid \mathbf{s}) \in \mathcal{P}^{+}(\mathcal{A})$, aperiodic since any state $\mathbf{s} \in \mathcal{T}$ can be reached in either $|\mathbf{s}|$ or $|\mathbf{s}|+1$ steps.
}

A couple of points about the notation. For any string $\mathbf{u}$, not just strings in $\mathcal{T}$, we will use stationary probability of $\mathbf{u}, \pi(\mathbf{u})$, to mean $p_{\mathcal{T}, q}\left(Y_{1}^{|\mathbf{u}|}=\mathbf{u}\right)$. If $\mathbf{s} \in \mathcal{T}$, our notation is redundant - the transition probability $q(a \mid \mathbf{s})$ and $p_{\mathcal{T}, q}(a \mid \mathbf{s})$ are synonymous. However, if $\mathbf{u} \notin \mathcal{T}$, we will only use $p_{\mathcal{T}, q}(a \mid \mathbf{u})$ and avoid using $q(a \mid \mathbf{u})$.

\section{Difficulties in Estimation}

It is quite possible all strings in a finite sample, no matter how large, have arbitrarily small mass under the stationary distribution. We illustrate this in Example 2 below. Our results, particularly Theorem 7 incorporates this phenomenon, and we try to provide the best results despite this apparent difficulty.

Example 2. Let $\mathcal{A}=\{0,1\}$ and $\mathcal{T}=\{0,1\}$ with $q(1 \mid 1)=$ $1-\epsilon$, and $q(1 \mid 0)=\frac{\epsilon}{m}$. For $\epsilon>0$ and a constant $m \in \mathbb{R}$ with $m>\epsilon$, this model represents a stationary ergodic Markov process $p_{\mathcal{T}, q}$ with stationary distributions $\pi(1)=\frac{1}{m+1}, \pi(0)=$ $\frac{m}{m+1}$. Note that $\pi(1)$ can be arbitrarily small for sufficiently large $m$.

Now suppose we have a length- $n$ sample with $\epsilon \ll 1 / n$. If we start from 1, with high probability we see a sequence of $n$ consecutive 1's. For instance, if $\epsilon=1 / n^{j}$ for some $j \geq 2$, then with probability $\geq 1-1 / n$ under $p_{\mathcal{\tau}, q}$, we see a sequence of $n$ consecutive 1's. Clearly, the stationary probability of any sequence of 1 's is $\leq \frac{1}{m+1}$, and this can be made arbitrarily small by choosing $m$ large enough.

The next example illustrates one pitfall of having no bound on the memory. We therefore require that dependencies die down by requiring that conditional probabilities satisfy (6) in Section VIII

Example 3. Let $\mathcal{T}=\mathcal{A}^{k}$ denote a full tree with depth $k$ and $\mathcal{A}=\{0,1\}$. Assume that $q\left(1 \mid 0^{k}\right)=2 \epsilon$ and $q\left(1 \mid 10^{k-1}\right)=1-\epsilon$ with $\epsilon>0$, and let $q(1 \mid \mathbf{s})=\frac{1}{2}$ (where $0^{k}$ indicates a string with $k$ consecutive zeros) for all other $\mathbf{s} \in \mathcal{T}$. Let $p_{\mathcal{T}, q}$ represent the stationary ergodic Markov process associated with this model. Observe that stationary probability of being in state $0^{k}$ is $\frac{1}{2^{k+1}-1}$ while all other states have stationary probability $\frac{2}{2^{k+1}-1}$. Let $Y_{1}^{n}$ be a realization of this process with initial state $1^{k} \preceq Y_{-\infty}^{0}$. Suppose $k \gg \omega(\log n)$. $\rrbracket^{4}$ With high probability we will never find a string of $k-1$ zeros among $n$ samples, and every bit is generated with probability

\footnotetext{
${ }^{4}$ A function $f_{n}=\omega\left(g_{n}\right)$ if $\lim _{n \rightarrow \infty} f_{n} / g_{n}=\infty$.
}

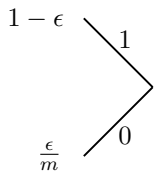

Fig. 2. Markov processes in Example 2 with stationary probabilities $\pi(1)=$ $\frac{1}{m+1}$ and $\pi(0)=\frac{m}{m+1}$. 


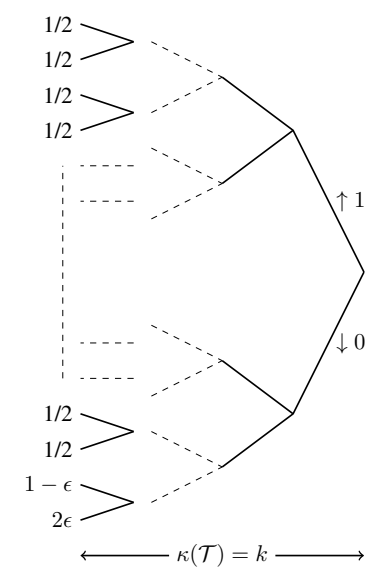

Fig. 3. Markov process in Example 3 With high probability, we cannot distinguish $p_{\mathcal{T}, q}$ from an i.i.d. Bernoulli(1/2) process if the sample size $n$ satisfies $k \gg \omega(\log n)$.

$1 / 2$. Thus with samples of size $n$, no matter how large $n$ may be, with high probability we cannot distinguish certain longmemory processes from even an i.i.d. Bernoulli(1/2) process.

The third example illustrates complications arising from mixing properties while estimating stationary probabilities.

Example 4. Let $\mathcal{A}=\{0,1\}$ and $\mathcal{T}=\{0,1\}$ with $q(1 \mid 1)=$ $1-\epsilon$, and $q(1 \mid 0)=\epsilon$. For $\epsilon>0$, this model represents a stationary ergodic Markov process with stationary distributions $\pi(1)=\frac{1}{2}, \pi(0)=\frac{1}{2}$. Let $\mathcal{T}^{\prime}=\{0,1\}$ with $q^{\prime}(1 \mid 1)=1-$ $\epsilon, q^{\prime}(1 \mid 0)=2 \epsilon$. Similarly, for $\epsilon>0$ this model represents a stationary ergodic Markov process with stationary distributions $\pi^{\prime}(1)=\frac{2}{3}, \pi^{\prime}(0)=\frac{1}{3}$.

Suppose we have a length- $n$ sample and suppose $\epsilon \ll 1 / n$. If we start from 1 (or 0 ), both models will yield a sequence of $n$ 1's (or 0's) with high probability. Therefore, the length $n$ samples from the two sources look identical. Hence no estimator could distinguish between these two models with high probability if $\epsilon \ll o(1 / n)$, and therefore no estimator can obtain their stationary probabilities either.

Finally there is, of course, no guarantee that the counts of

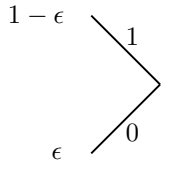

(a)

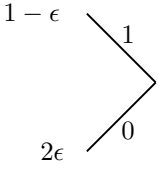

(b)
Fig. 4. Markov processes in Example 4 with stationary probabilities (a) $\pi(1)=\pi(0)=\frac{1}{2}$ (b) $\pi^{\prime}(1)=\frac{2}{3}, \pi^{\prime}(0)=\frac{1}{3}$. Given a sample with size $n$ with $\epsilon \ll o(1 / n)$, we cannot distinguish between these two models.

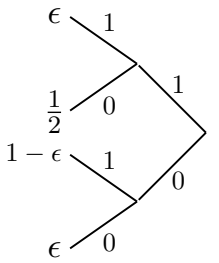

(a)

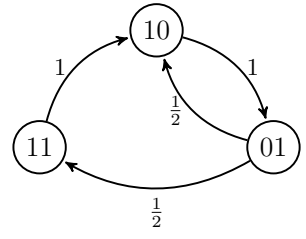

(b)
Fig. 5. (a) Markov in Example 5 (b) Same process when $\epsilon=0$.

short strings are more amenable to interpretation than longer ones in a long-memory, slow mixing process.

Example 5. Let $\mathcal{T}=\{11,01,10,00\}$ with $q(1 \mid 11)=\epsilon$, $q(1 \mid 01)=\frac{1}{2}, q(1 \mid 10)=1-\epsilon, q(1 \mid 00)=\epsilon$. If $\epsilon>0$, then $p_{\mathcal{T}, q}$ is a stationary ergodic binary Markov process. Let $\pi$ denote the stationary distribution of this process. A simple computation shows that $\pi(11)=\frac{1}{7-6 \epsilon}, \pi(01)=\frac{2-2 \epsilon}{7-6 \epsilon}, \pi(10)=\frac{2-2 \epsilon}{7-6 \epsilon}$ and $\pi(00)=\frac{2-2 \epsilon}{7-6 \epsilon}$, and $\pi(1)=\frac{1}{7-6 \epsilon}+\frac{2-2 \epsilon}{7-6 \epsilon}=\frac{3-2 \epsilon}{7-6 \epsilon}$ and $\pi(0)=\frac{2-2 \epsilon}{7-6 \epsilon}+\frac{2-2 \epsilon}{7-6 \epsilon}=\frac{4-4 \epsilon}{7-6 \epsilon}$.

Suppose we have a length $n$ sample. If $\epsilon \ll \frac{1}{n}$, then $\pi(1) \approx$ $\frac{3}{7}$ and $\pi(0) \approx \frac{4}{7}$. If the initial state belongs to $\{11,01,10\}$, the state 00 will not be visited with high probability in $n$ samples, and it can be seen that the counts of 1 or 0 will not be near the stationary probabilities $\pi(1)$ or $\pi(0)$. For this sample size, the process effectively acts like the irreducible, aperiodic Markov chain in Fig. 5. (b) which can be shown to be fast mixing. Therefore the counts of 01,10 and 11 approach the stationary probabilities of the chain in Fig. 5. (b), namely $\frac{\pi(01)}{\pi(1)+\pi(10)}$, $\frac{\pi(10)}{\pi(1)+\pi(10)}$, and $\frac{\pi(11)}{\pi(1)+\pi(10)}$, much quicker than the counts of 1 and 0 will approach $\pi(1)$ or $\pi(0)$. Indeed, this observation guides our search for results in Section XII.

\section{Summary OF Results}

We observe a length $n$ sample from a stationary, ergodic, $\mathcal{A}$-ary Markov source $p_{\mathcal{T}, q}$, where both $\mathcal{T}$ and $q(\mathcal{T})$ are unknown. Using this sample, we want (i) to approximate as best as possible, the parameter set $q(\mathcal{T})$ (ii) the stationary probabilities $\pi(\mathbf{s})$ of strings $\mathbf{s} \in \mathcal{T}$, and (iii) to estimate or at least obtain heuristics of the entropy rate of the process.

Two distinct problems complicate estimation of $q(\mathcal{T})$ and the stationary probabilities. First is the issue that the memory may be too long to handle-in fact, if the source has long enough memory it may not be possible, with $n$ samples, to distinguish the source even from a memoryless source (Example 3). Second, even if the source has only one bit of memory, it may be arbitrarily slow mixing (Example 4). No matter what $n$ is, there will be sources against which our estimates perform very poorly.

Given $Y_{-\infty}^{0}$, we get the sample sequence $Y_{1}^{n}$ from the (unknown) model, namely $Y_{i}$ is generated with probabilities $q\left(Y_{i} \mid \mathbf{c}_{\mathcal{T}}\left(Y_{-\infty}^{i-1}\right)\right)$. Since we do not know $\mathcal{T}$, a natural way to proceed is to estimate conditional probabilities of form $p_{\mathcal{T}, q}\left(Y_{1} \mid \mathbf{u}\right)$, where $\mathbf{u} \in \mathcal{A}^{k_{n}}$ are strings of a given length $k_{n}$. Thus, we obtain a potentially coarser model with states 
$\tilde{\mathcal{T}}=\mathcal{A}^{k_{n}}$ for some known $k_{n}$. With the benefit of hindsight, we take $k_{n}=\mathcal{O}(\log n)^{5}$ and write $k_{n}=\alpha_{n} \log n$ for some function $\alpha_{n}=\mathcal{O}(1)$. This scaling of $k_{n}$ also reflects well known conditions for consistency of estimation of Markov processes in [24].

For convenience, we rephrase the above problem by defining an aggregation of a Markov process in Section VII at depth $k_{n}$. The aggregation of the true model can be thought of a coarse approximation of the true model- the aggregated model has memory $k_{n}$ and (unknown) parameters associated with $\mathbf{u} \in$ $\mathcal{A}^{k_{n}}, \tilde{q}(a \mid \mathbf{u})$, set to $p_{\mathcal{T}, q}(a \mid \mathbf{u})$. We denote the aggregated model by $p_{\tilde{\mathcal{T}}, \tilde{q}}($ with states $\tilde{\mathcal{T}}$ and parameters $\tilde{q}(\tilde{\mathcal{T}})$ ). In Proposition 1 . we show that the entropy rate corresponding to the aggregated model $p_{\tilde{\mathcal{T}}, \tilde{q}}$ is an upper bound on the entropy rate of the true model $p_{\mathcal{T}, q}$.

The catch is that we do not get to see observations corresponding to the aggregated model. We need to estimate the aggregated model $p_{\tilde{\mathcal{\tau}} \tilde{q}}$ using the observations from the true, underlying model $p_{\mathcal{\tau}, q}$. Therefore the task is not the same as estimating a model with memory $k_{n}$.

Naive estimates: To obtain the parameters of the aggregated process, $\tilde{q}(\tilde{\mathcal{T}})$, suppose we use a naive estimator that proceeds as though the sample was in fact generated from $p_{\tilde{\mathcal{T}}, \tilde{q}}$. Namely the naive estimator is based on the premise that the subsequence of symbols in the sample following any $\mathbf{w} \in \tilde{\mathcal{T}}$ is i.i.d.. Consider the following illustration.

Suppose the binary Markov sample is 1101010100 . Let $Y_{-\infty}^{0}=\cdots 00$. We want to estimate the aggregated parameters at depth 2-the conditional probability that $a$ follows given a two bit string. In particular, say we want the aggregated parameters associated with string 10, $\tilde{q}(a \mid 10)=p_{\mathcal{T}, q}(a \mid 10)$. The subsequence following 10 in the sample is 1110 . Then the naive estimate of $\tilde{q}(1 \mid 10)$, denoted by $\hat{q}(1 \mid 10)$, is $3 / 4$ and the naive estimate of $\tilde{q}(0 \mid 10)$, denoted by $\hat{q}(0 \mid 10)$, is $1 / 4$.

There are two ways the naive estimate may still work. (i) The counts of 101 and 100 reflect their stationary probabilities, which automatically means that the count of 10 represents its stationary probability as well. (ii) The subsequence 1110 that follows 10 is i.i.d.. Case (i) is not valid since we have not assumed that the source has mixed. Case (ii) would only hold if the string $10 \in \mathcal{T}$ as well or if the sample was from the aggregated source at depth 2-neither assumption is justified at this point. Therefore, in general there is no reason why the naive approach even makes sense. However, assuming dependencies die down as below, we show that certain naive estimates still capture the conditional probabilities accurately.

Dependencies die down: However, it is reasonable given our motivation that the influence of prior symbols dies down as we look further into the past- the incremental value of an additional page in browser history diminishes when the amount of history we already have access to increases.

We formalize this notion in Section VIII with a function $d(i)$ that controls how symbols $i$ locations apart can influence each other, and require $\sum_{i>1} d(i)<\infty$. Let $\mathcal{M}_{d}$ be the set of all models $p_{\mathcal{T}, q}$ that satisfy for all $\mathbf{u} \in \mathcal{A}^{*}$ and all $b, b^{\prime} \in \mathcal{A}$

\footnotetext{
${ }^{5}$ A function $f_{n}=\mathcal{O}\left(g_{n}\right)$ if $\exists n_{0} \in \mathbb{N}$ and $\exists M>0$ such that $f_{n} \leq M g_{n}$ for $n \geq n_{0}$.
}

and for all $a \in \mathcal{A}$.

$$
\left|\frac{p_{\mathcal{T}, q}(a \mid b \mathbf{u})}{p_{\mathcal{T}, q}\left(a \mid b^{\prime} \mathbf{u}\right)}-1\right| \leq d(|\mathbf{u}|) .
$$

It can be easily shown that the mutual information between bits $i$ apart, conditioned on all bits between them, is upper bounded by $\log (1+d(i))$. Note that there is no bound on the memory of models in $\mathcal{M}_{d}$. Moreover, the function $d$ does not constrain mixing properties of the processes as we show in Section VIII

Conditional probabilities: For sources $p_{\tau_{\mathcal{\alpha}} q} \in \mathcal{M}_{d}$, we show how to obtain (from the data sample) $G \subseteq \tilde{\mathcal{T}}$, a set of good states $\sqrt{6}$ or good length- $k_{n}$ strings (Definition 4) from sample sequence. These are strings that will be amenable to concentration results, and hence the adjective "good". These results do not depend on empirical counts of strings being near their stationary probabilities, nor do they require that the subsequence following a string $\mathrm{w} \in \tilde{\mathcal{T}}$ be i.i.d.

Main Result 1: In Theorem 2, we show that with probability (under the underlying unknown $p_{\mathcal{T}, q}$ ) $\geq 1-\frac{1}{2^{\mid \mathcal{A}^{k} k_{n}+1} \log n}$ (conditioned on any past $Y_{-\infty}^{0}$ ), for all states $\mathbf{w} \in \tilde{\mathcal{T}}$ simultaneously

$$
\|\tilde{q}(\cdot \mid \mathbf{w})-\hat{q}(\cdot \mid \mathbf{w})\|_{1} \leq 2 \sqrt{\frac{(\ln 2)\left(|\mathcal{A}|^{k_{n}+1} \log n+n \delta_{k_{n}}\right)}{N_{\mathbf{w}}}} .
$$

Here, $\delta_{k_{n}}=\sum_{i \geq k_{n}} d(i), N_{\mathbf{w}}$ is the number of occurrences of string $\mathbf{w}$ in the sample and $\hat{q}(. \mid \mathbf{w})$ is the naive estimator of $\tilde{q}(. \mid \mathbf{w})$ as described above. Note that (2) automatically yields sharper estimates for those strings $\mathbf{w}$ whose counts are larger.

For example, if $d(i)=\gamma^{i}$ for some $0<\gamma<\frac{1}{2}$, then $\delta_{k_{n}}=\frac{\gamma^{k_{n}}}{1-\gamma}$. By choosing $k_{n}=\gamma \log n$, the accuracy of $\hat{q}(\cdot \mid \mathbf{w})$ is $\Theta\left(\sqrt{\frac{n^{1+\gamma \log \gamma}}{N_{\mathrm{w}}}}\right)$. In particular, for a strings appearing at least $\frac{n}{\log n}$ times, the accuracy of estimation in (2) is better than $\Theta\left(\sqrt{n^{\gamma \log \gamma \log n}}\right)$.

The above estimation result is built on two facts: (i) dependencies dying down and (ii) universal compression results on $\mathcal{M}_{d}$ built on the fact that length- $n$ sequences generated by Markov sources with memory $k_{n}$ can be universally compressed if $k_{n}=\mathcal{O}(\log n)$.

A related curiosity arises due to the fact that the above result does not depend on empirical frequencies being close to stationary probabilities. The result above is sometimes tight for strings $\mathbf{w}$ while being vacuous for their suffixes $\mathbf{w}^{\prime}$. For example, it could be that we estimate parameters associated with a string $\mathbf{w}$ of length $\Theta(\log n)$ (say $\mathbf{w}$ is a string of ten 0 's) but not those associated with $\mathbf{w}^{\prime}$ where $\mathbf{w}^{\prime} \preceq \mathbf{w}$ (say $\mathbf{w}^{\prime}$ is a string of five 0's). Finally, since the above result is what can be obtained without any knowledge of mixing properties, (2) could be strengthened using other arguments in literature in cases where we may know that empirical counts reflect stationary probabilities.

Stationary probabilities of strings: In general, stationary probabilities of strings can be a very sensitive function of the transition probabilities. We now have the approximate

\footnotetext{
${ }^{6}$ Strings in $\tilde{\mathcal{T}}$ may not be states of $p_{\mathcal{T}, q}$, but we abuse this notation for convenience.
} 
transition probabilities associated with strings in $\tilde{G}$. With this little bit of information we have gleaned from the sample, can we even hope to say anything about stationary probabilities of w $\in \tilde{G}$ ? How then do we interpret the empirical counts $N_{\mathrm{w}}$ of various strings $\mathbf{w}$ ?

To answer this question, we calculate a parameter $\eta_{\tilde{G}}$ in (8) which resembles the Dobrushin's ergodicity coefficient of Markov processes, but which can be estimated well using (2). Suppose $\left\{\delta_{i}\right\}_{i \geq 1}$ is summable as well, and let $\Delta_{j}=\sum_{i \geq j} \delta_{i}$.

Main Result 2: In Theorem 7 we show under a minor technical condition that for all $t>0, Y_{-\infty}^{0}$ and $\mathbf{w} \in \tilde{G}$ the counts of $\mathbf{w}$ in the sample, $N_{\mathbf{w}}$, concentrates though not necessarily around $\pi(\mathbf{w})$. We show that

$$
p_{\mathcal{T}, q}\left(\left|N_{\mathbf{w}}-\tilde{n} \frac{\pi(\mathbf{w})}{\pi(\tilde{G})}\right| \geq t \mid Y_{-\infty}^{0}\right) \leq 2 \exp \left(-\frac{(t-\mathcal{B})^{2}}{2 \tilde{n} \mathcal{B}^{2}}\right),
$$

where $\mathcal{B} \approx 4 \max \left\{\ell_{n}, k_{n}\right\} /\left[\eta_{\tilde{G}}^{k_{n}}\left(1-\Delta_{k_{n}}\right)\right]$. Here, $\ell_{n}$ is the smallest integer such that $\Delta_{\ell_{n}} \leq \frac{1}{n}, \tilde{n}$ is the total count of good states in the sample and $\pi$ denotes the stationary distribution of $p_{\mathcal{T}, q}$. Note once again that $p_{\mathcal{T}, q}$ is the probability law under the underlying unknown model in $\mathcal{M}_{d}$. The above estimation result (3) uses a coupling argument [6] to bound martingale differences of a natural Doob martingale construction in Section XII.

When dependencies $d(i)$ decay exponentially, we will have $\ell_{n}=\Theta(\log n)$. Suppose $\tilde{n}=\mathcal{O}(n)$ and we want our confidence to approach 1 polynomially in $n$. If $\eta_{\tilde{G}}$ specified in Section XII is $\Theta(1)$ and sufficiently large, it implies reasonable mixing within the good states. In this case, there will be $0<\beta<\frac{1}{2}$ such that for all $\mathbf{w} \in \tilde{G}, N_{w} / \tilde{n}$ is within $\frac{(\log n)^{2}}{n^{1 / 2-\beta}}$ from the ratio $\pi(\mathbf{w}) / \pi(\tilde{G})$ with the required confidence. In case $\eta_{\tilde{G}}$ turns out to be too small to yield good deviation bounds, one can either shrink $\tilde{G}$ to include a subset of states that mix well, or move to smaller values for $k_{n}$ (but still scaling as $\Theta(\log n))$.

To summarize, note that $k_{n}, \Delta_{k_{n}}$ and $\ell_{n}$ (the later two related to how fast dependencies die down) are known $a-$ priori. But $\tilde{n}$ is a random variable found from the sample. So is $\eta_{\tilde{G}}$, but one that can be well estimated from the sample using (2). The result (3) is a natural deviation bound where the confidence (right side of (3) ) is a random variable generated from the model $p_{\mathcal{T}, q}$, but one that can be well estimated from the sample (the confidence in (3) is a decreasing function of $\eta_{\tilde{G}}$ ). To use (3) when confronted with a sample, we lower bound $\eta_{\tilde{G}}$ by $\bar{\eta}_{\tilde{G}}$ with confidence $\geq 1-\frac{1}{2^{|\mathcal{A}|^{k} n+1} \log n}$ using (2) to conservatively obtain a further upper bound on the left side of (3).

Remark All logarithms are base 2. We use bold font $\mathbf{w}$ or $\mathbf{s}$ for strings. Typically $\mathbf{s}$ is a generic state or context of a process, while w is used for a "good" state as a mnemonic. A subscript s usually refers to an instance of the Markov process whose past corresponds to $\mathbf{s}$-for example, $N_{\mathbf{s}}$ (for the count of $\mathbf{s}$ in the sample-the number of times the sample had $\mathbf{s}$ as its immediate past).

\section{BACKGROUND}

\section{A. Context tree weighting}

Context tree weighting is a universal data compression algorithm for Markov sources [14], [21] that captures several insights on how Markov processes behave in non-asymptotic regimes. Let $y_{1}^{n}$ be sequence of symbols from an alphabet $\mathcal{A}$. Let $\hat{\mathcal{T}}=\mathcal{A}^{K}$ for some positive integer $K$. For all $\mathrm{s} \in \hat{\mathcal{T}}$ and $a \in \mathcal{A}$, let $n_{\mathbf{s} a}$ be the number of $a$ 's which appear exactly after the string $\mathbf{s}$ in $y_{1}^{n}$. The depth- $K$ context tree weighting constructs a distribution $p_{c}$ satisfying 7

$$
p_{c}\left(y_{1}^{n} \mid y_{-K+1}^{0}\right) \geq 2^{-|\mathcal{A}|^{K+1} \log n} \prod_{\mathbf{s} \in \hat{\mathcal{T}}} \prod_{a \in \mathcal{A}}\left(\frac{n_{\mathbf{s} a}}{\sum_{a \in \mathcal{A}} n_{\mathbf{s} a}}\right)^{n_{\mathbf{s} a}} .
$$

The above inequality is not the strongest, but its form is convenient for use. Note that no Markov source with memory $K$ could have given a higher probability to $y_{1}^{n}$ than

$$
\prod_{\mathbf{s} \in \hat{\mathcal{T}}} \prod_{a \in \mathcal{A}}\left(\frac{n_{\mathbf{s} a}}{\sum_{a \in \mathcal{A}} n_{\mathbf{s} a}}\right)^{n_{\mathbf{s} a}} .
$$

So, if $|\mathcal{A}|^{K} \log n=o(n)$, then $p_{c}$ underestimates any memory$K$ Markov probability by only a subexponential factor. Therefore, $K=\mathcal{O}(\log n)$ is going to be the case of particular interest.

\section{B. Coupling for Markov processes}

We adopt the coupling [6] technique in Section XII to estimate stationary probabilities. Coupling is an elegant approach to interpret counts of certain strings in a sample. Let $p_{\mathcal{T}, q}$ be our Markov source generating sequences from an alphabet $\tilde{\mathcal{A}}$.

A coupling $\mu$ for $p_{\mathcal{T}, q}$ is a special kind of joint probability distribution on the sequences $\left\{Y_{m}, \bar{Y}_{m}\right\}_{m \geq 1}$ where $Y_{m} \in \tilde{\mathcal{A}}$ and $\bar{Y}_{m} \in \tilde{\mathcal{A}}$. $\mu$ has to satisfy the following property: individually taken, the sequences $\left\{Y_{m}\right\}$ and $\left\{\bar{Y}_{m}\right\}$ have to be faithful evolutions of $p_{\mathcal{\tau}, q}$. Specifically, for $m \geq 0$, we want

$$
\begin{aligned}
\mu\left(Y_{m+1} \mid Y_{-\infty}^{m}, \bar{Y}_{-\infty}^{m}\right) & =p_{\mathcal{T}, q}\left(Y_{m+1} \mid Y_{-\infty}^{m}\right) \\
& =p_{\mathcal{T}, q}\left(Y_{m+1} \mid \mathbf{c}_{\mathcal{T}}\left(Y_{-\infty}^{m}\right)\right),
\end{aligned}
$$

and similarly for $\left\{\bar{Y}_{m}\right\}$.

In the context of this paper, we think of $\left\{Y_{m}\right\}$ and $\left\{\bar{Y}_{m}\right\}$ as copies of $p_{\mathcal{T}, q}$ that were started with two different states $\mathbf{s}, \mathbf{s}^{\prime} \in \mathcal{T}$ respectively, but the chains evolve jointly as $\mu$ instead of independently. For any $r$ and $\mathbf{w} \in \tilde{\mathcal{A}}^{r}, N_{\mathbf{w}}$ (respectively $\bar{N}_{\mathbf{w}}$ ) is the number of times $\mathbf{w}$ forms the context of a symbol in a length- $n$ time frame, $\left\{Y_{i}\right\}_{i=1}^{n}$ given $Y_{-\infty}^{0}$

\footnotetext{
${ }^{7}$ Note that the bound holds for $n \geq 2$.
} 
(respectively $\left\{\bar{Y}_{i}\right\}_{i=1}^{n}$ given $\bar{Y}_{-\infty}^{0}$ ). Then, for any $\mu^{8}$

$$
\begin{aligned}
& \left|\mathbb{E}_{p_{\mathcal{T}, q}}\left[N_{\mathbf{w}} \mid Y_{-\infty}^{0}\right]-\mathbb{E}_{p_{\mathcal{T}, q}}\left[\bar{N}_{\mathbf{w}} \mid \bar{Y}_{-\infty}^{0}\right]\right| \\
& =\left|\sum_{i=1}^{n} \mathbb{E}_{\mu}\left[\mathbb{1}\left(\mathbf{c}_{\tilde{\mathcal{A}}^{r}}\left(Y_{-\infty}^{i}\right)=\mathbf{w}\right)-\mathbb{1}\left(\mathbf{c}_{\tilde{\mathcal{A}}^{r}}\left(\bar{Y}_{-\infty}^{i}\right)=\mathbf{w}\right)\right]\right| \\
& \leq \sum_{i=1}^{n}\left|\mathbb{E}_{\mu}\left[\mathbb{1}\left(\mathbf{c}_{\tilde{\mathcal{A}}^{r}}\left(Y_{-\infty}^{i}\right)=\mathbf{w}\right)-\mathbb{1}\left(\mathbf{c}_{\tilde{\mathcal{A}}^{r}}\left(\bar{Y}_{-\infty}^{i}\right)=\mathbf{w}\right)\right]\right| \\
& \leq \sum_{i=1}^{n} \mu\left(\mathbf{c}_{\tilde{\mathcal{A}}^{r}}\left(Y_{-\infty}^{i}\right) \neq \mathbf{c}_{\tilde{\mathcal{A}}^{r}}\left(\bar{Y}_{-\infty}^{i}\right)\right),
\end{aligned}
$$

where the first equality follows from (4).

The art of a coupling argument stems from the fact that $\mu$ is completely arbitrary apart from having to satisfy (4). If we can find any $\mu$ such that the chains coalesce, namely $\mu\left(\mathbf{c}_{\tilde{\mathcal{A}}^{r}}\left(Y_{-\infty}^{i}\right) \neq \mathbf{c}_{\tilde{\mathcal{A}}^{r}}\left(\bar{Y}_{-\infty}^{i}\right)\right)$ becomes small as $i$ increases, then we know that $\mathbb{E}_{p_{\mathcal{T}, q}}\left[N_{\mathbf{w}} \mid Y_{-\infty}^{0}\right]$ cannot differ too much from $\mathbb{E}_{p_{\mathcal{T}, q}}\left[\bar{N}_{\mathbf{w}} \mid \bar{Y}_{-\infty}^{0}\right]$.

Now if, in addition, such a coalescence holds no matter what $\bar{Y}_{-\infty}^{0}$ is, we could then pick $\bar{Y}_{-|\mathcal{T}|}^{0}$ according to the stationary distribution of $p_{\mathcal{T}, q}$. Then $\bar{N}_{\mathbf{w}}$ would be close to the stationary count of $\mathbf{w}$, and from the coupling argument above, so is $N_{\mathbf{w}}$. For tutorials, see e.g., [41], [5], [42].

\section{Model Aggregation}

\section{A. Entropy Rate}

The entropy rate of a stationary Markov process $p_{\mathcal{T}, q}$, denoted by $\mathcal{H}_{\mathcal{T}}$, is defined as [43]

$$
\mathcal{H}_{\mathcal{T}}=-\sum_{\mathbf{s} \in \mathcal{T}} \pi(\mathbf{s}) \sum_{a \in \mathcal{A}} q(a \mid \mathbf{s}) \log q(a \mid \mathbf{s}) \stackrel{\text { def }}{=} \sum_{\mathbf{s} \in \mathcal{T}} \pi(\mathbf{s}) \mathcal{H}_{\mathbf{s}} .
$$

\section{B. Aggregations}

Since the memory is unknown a-priori, a natural approach, known to be consistent, is to use a potentially coarser model with depth $k_{n}$. Here, $k_{n}$ increases logarithmically with the sample size $n$, and reflects [24] well known results on consistent estimation of Markov processes. We show that coarser models formed by properly aggregating states of the original context tree model are useful in upper bounding entropy rates of the true process.

Definition 2. Suppose $\tilde{\mathcal{T}}=\mathcal{A}^{k}$ for some positive integer $k$. The aggregation of $p_{\mathcal{T}, q}$ at level $k$, denoted by $p_{\tilde{\mathcal{\tau}}, \tilde{q}}$, is a stationary Markov process with state transition probabilities given by

$$
\tilde{q}(a \mid \mathbf{s})=p_{\mathcal{T}, q}(a \mid \mathbf{s})=\frac{\sum_{\mathbf{v} \in \mathcal{T}_{\mathbf{s}}} \pi(\mathbf{v}) q(a \mid \mathbf{v})}{\sum_{\mathbf{v}^{\prime} \in \mathcal{T}_{\mathbf{s}}} \pi\left(\mathbf{v}^{\prime}\right)},
$$

for all $\mathbf{s} \in \tilde{\mathcal{T}}$ and $a \in \mathcal{A}$, where $\pi$ is the stationary distribution associated with $p_{\mathcal{T}, q}$. Using Observation 11, wolog, no matter

$$
\begin{aligned}
& \text { For any event } E \text {, the indicator function is defined as } \\
& \qquad \mathbb{1}(E)=\left\{\begin{array}{lc}
1 & \text { if } E \text { holds } \\
0 & \text { otherwise. }
\end{array}\right.
\end{aligned}
$$

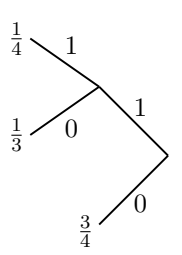

(a)

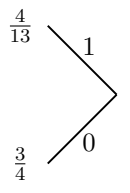

(b)
Fig. 6. (a) Markov process in Example 6 (b) Aggregated model at depth 1. From Observation 1, the model on the left can be reparameterized to be a complete tree at any depth $\geq 2$. We can hence ask for its aggregation at any depth. Aggregations of the above model on the left at depths $\geq 2$ will hence be the model itself.

what $\tilde{\mathcal{T}}$ is, we will assume $p_{\mathcal{T}, q}$ has states $\mathcal{T}$ such that $\tilde{\mathcal{T}} \preceq \mathcal{T}$.

Example 6. This example illustrates the computations in Definition above. Let $p_{\mathcal{T}, q}$ be a binary Markov process with $\mathcal{T}=\{11,01,0\}$ and $q(1 \mid 11)=\frac{1}{4}, q(1 \mid 01)=\frac{1}{3}, q(1 \mid 0)=$ $\frac{3}{4}$. For this model, we have $\pi(11)=\frac{4}{25}, \pi(01)=\frac{9}{25}$ and $\pi(0)=\frac{12}{25}$. Fig. 6 (b) shows an aggregated process $p_{\tilde{\tau}, \tilde{q}}$ with $\tilde{\mathcal{T}}=\{1,0\}$. Notice that $\tilde{q}(1 \mid 1)=\left(\frac{4}{25} \frac{1}{4}+\frac{9}{25} \frac{1}{3}\right) /\left(\frac{4}{25}+\frac{9}{25}\right)=\frac{4}{13}$ and $\tilde{q}(1 \mid 0)=\frac{3}{4}$.

Lemma 1. Let $p_{\mathcal{T}, q}$ be a stationary Markov process with stationary distribution $\pi$. If $p_{\tilde{\mathcal{T}}, \tilde{q}}$ aggregates $p_{\mathcal{T}, q}$ then it has a unique stationary distribution $\tilde{\pi}$ and for every $\mathbf{w} \in \tilde{\mathcal{T}}$

$$
\tilde{\pi}(\mathbf{w})=\sum_{\mathbf{v} \in \mathcal{T}_{\mathbf{w}}} \pi(\mathbf{v})
$$

Proof See Appendix A

\section{Upper Bound}

Suppose $p_{\tilde{\mathcal{T}}, \tilde{q}}$ aggregates $p_{\mathcal{T}, q}$, and let the entropy rate of the aggregated process be $\mathcal{H}_{\tilde{\mathcal{T}}}$. Then,

Proposition 1. $\mathcal{H}_{\mathcal{T}} \leq \mathcal{H}_{\tilde{\mathcal{T}}}$.

Proof See Appendix B

Remark In this paper, we are particularly concerned with the slow mixing regime. As our results will show, in general it is not possible to obtain a simple upper bound on the entropy rate using the data (given a particular starting state) and taking recourse to the Proposition above. Instead, we introduce the partial entropy rate that can be reliably obtained from the data

$$
\mathcal{H}_{\tilde{G}}=\sum_{\mathbf{w} \in \tilde{G}} \frac{\pi(\mathbf{w})}{\pi(\tilde{G})} \mathcal{H}_{\mathbf{w}},
$$

where $\tilde{G} \subseteq \tilde{\mathcal{T}}$ will be a set of good states that we show how to identify. Recall that $\pi(\tilde{G})$ may be arbitrarily small in a finite sample, hence we must expect to compute $\mathcal{H}_{\tilde{G}}$ and not $\pi(\tilde{G}) \mathcal{H}_{\tilde{G}}$. The partial entropy rate is not necessarily an upper bound, but in slow mixing cases it is sometimes the best heuristic possible. We systematically handle the entropy rates of slow mixing processes using the estimation results below in different paper. 


\section{DEPENDENCIES IN THE MODEL}

As noted before in Example 3, if the dependencies could be arbitrary in a Markov process, we will not estimate the model accurately no matter how large the sample is. Keeping in mind Observation 1, we formalize dependencies dying down by means of a function $d: \mathbb{Z}^{+} \rightarrow \mathbb{R}^{+}$with $\sum_{i=1}^{\infty} d(i)<\infty$.

Let $\mathcal{M}_{d}$ be the set of all models $p_{\mathcal{T}, q}$ that satisfy for all $\mathbf{u} \in \mathcal{A}^{*}$ and all $b, b^{\prime} \in \mathcal{A}$ and for all $a \in \mathcal{A}$.

$$
\left|\frac{p_{\mathcal{T}, q}(a \mid b \mathbf{u})}{p_{\mathcal{T}, q}\left(a \mid b^{\prime} \mathbf{u}\right)}-1\right| \leq d(|\mathbf{u}|) \text {. }
$$

Note that our collection $\mathcal{M}_{d}$ has bounded memory iff there exists a finite $K$ such that $d(i)=0$ for all $i>K$.

Remark We emphasize that the restriction $\{d(i)\}_{i \geq 1}$ does not preclude slow mixing processes in $\mathcal{M}_{d}$-we clarify this point in following example. The restriction $\{d(i)\}_{i \geq 1}$ we use is related to the notion of "continuity rate" of stochastic processes used in [44], [34], [45], [33].

Example 7. Let $p_{\mathcal{T}, q}$ be a context tree model with $\mathcal{T}=$ $\{0,1\}$ and transition probabilities $q(1 \mid 1)=1-\epsilon, q(1 \mid 0)=\epsilon$ as in Fig. 4. (a). A simple calculation shows that the stationary probability of being at state 0 or 1 is $\frac{1}{2}$. Note that even with very strong restriction on $d$, namely $d(i)=0$ for $i \geq 1$, $(\mathcal{T}, q(\mathcal{T}))$ belongs to $\mathcal{M}_{d}$ regardless of the value of $\epsilon$.

While we do not need the notions of $\phi$-mixing and $\beta$ mixing coefficients for stationary stochastic processes [46] in the rest of the paper, we compute them for this example as an illustration that $\{d(i)\}_{i \geq 1}$ are unrelated to mixing.

Recall that the $j^{\prime}$ th $\phi$-mixing coefficient

$$
\begin{aligned}
\phi_{j} & \geq \max _{Y_{0} \in\{0,1\}} \max _{Y_{j} \in\{0,1\}}\left|p_{\mathcal{T}, q}\left(Y_{j} \mid Y_{0}\right)-p_{\mathcal{T}, q}\left(Y_{j}\right)\right| \\
& \geq\left|p_{\mathcal{T}, q}\left(Y_{j}=1 \mid Y_{0}=1\right)-p_{\mathcal{T}, q}\left(Y_{j}=1\right)\right| \\
& \geq p_{\mathcal{T}, q}\left(Y_{j}=1, Y_{j-1}=1, \cdots, Y_{1}=1 \mid Y_{0}=1\right) \\
& -p_{\mathcal{T}, q}\left(Y_{j}=1\right) \\
& =(1-\epsilon)^{j}-\frac{1}{2} .
\end{aligned}
$$

Similarly, the $j^{\prime}$ th $\beta$-mixing coefficient

$$
\begin{aligned}
\beta_{j} & \geq \mathbb{E}_{Y_{0}}\left[\max _{Y_{j} \in\{0,1\}}\left|p_{\mathcal{T}, q}\left(Y_{j} \mid Y_{0}\right)-p_{\mathcal{T}, q}\left(Y_{j}\right)\right|\right] \\
& =(1-\epsilon)^{j}-\frac{1}{2} .
\end{aligned}
$$

No matter what $\epsilon>0$ is, $p_{\mathcal{T}, q} \in \mathcal{M}_{d}$ even under stringent restriction $d(i)=0$ for $i \geq 1$. But, any $(\beta, \phi)$-mixing coefficient can be made arbitrarily as close to $\frac{1}{2}$ as possible by picking $\epsilon$ small enough. Therefore, the condition (6) we impose does not preclude slow mixing.

As mentioned in the last section, we will focus on set of the aggregated parameters at depth $k_{n}, \tilde{q}\left(\mathcal{A}^{k_{n}}\right)$ where $k_{n}=$ $\alpha_{n} \log n$. If $k_{n}$ is large enough, these aggregated parameters start to reflect the underlying parameters $q(\mathcal{T})$. Indeed, by using an elementary argument in Section $\mathrm{X}$ we will show that both the underlying and aggregated parameters will then be close to the empirically observed values for states that occur frequently enough — even though the sample we use comes from the true model $p_{\mathcal{T}, q}$ instead of the aggregated model $p_{\tilde{\mathcal{T}, \tilde{q}}}$.

Remark In the context of information aggregation on the Internet, suppose we have a browser history of the $i$ prior webpages (or more appropriately domains) visited. What is the incremental value of the $(i+1)^{\prime}$ th document in the past? It is reasonable to assume that as $i$ increases, the incremental value of another past document diminishes. It is easy to see using elementary arguments that in a Markov process $\left\{Y_{i}\right\}$, $i \in \mathbb{Z}$ within the class $\mathcal{M}_{d}$ that

$$
I\left(Y_{0}, Y_{i+1} \mid Y_{1}^{i}\right) \leq \log (1+d(i)),
$$

namely the function $d$ controls the incremental value of one additional data point in the history. It is interesting to note that not all Markov processes even in practical settings need to satisfy this observation (in fact problems in DNA folding specifically cannot make this assumption), but that this assumption is sufficient for statistical estimation problems to be well posed.

Proposition 2. Let $\{d(i)\}_{i \geq 1}$ be a sequence of real numbers such that there exists some $n_{0} \in \mathbb{N}$ for which, $0 \leq d(i) \leq 1$ for all $i \geq n_{0}$. Then, $\forall j \geq n_{0}$, we have

$$
1-\sum_{i \geq j} d(i) \leq \prod_{i \geq j}(1-d(i)) \leq \frac{1}{\prod_{i \geq j}(1+d(i))} .
$$

Proof See Appendix C

Proposition 3. Let $p_{\mathcal{T}, q} \in \mathcal{M}_{d}$. Suppose $p_{\tilde{\mathcal{T}}, \tilde{q}}$ aggregates $p_{\mathcal{T}, q}$ with $\tilde{\mathcal{T}}=\mathcal{A}^{k_{n}}$. If $\sum_{i \geq k_{n}} d(i) \leq 1$, then for all $\mathbf{w} \in \tilde{\mathcal{T}}$ and $a \in \mathcal{A}$

$$
\left(1-\sum_{i \geq k_{n}} d(i)\right) \max _{\mathbf{s} \in \mathcal{T}_{\mathbf{w}}} q(a \mid \mathbf{s}) \leq \tilde{q}(a \mid \mathbf{w}) \leq \frac{\min _{\mathbf{s} \in \mathcal{T}_{\mathbf{w}}} q(a \mid \mathbf{s})}{\left(1-\sum_{i \geq k_{n}} d(i)\right)} .
$$

Proof Recall that $\mathcal{T}_{\mathrm{w}}$ are nodes of $\mathcal{T}$ that are descendants of $\mathbf{w} \in \tilde{\mathcal{T}}$. See Appendix D.

\section{NAive Estimators}

Even in the slow mixing case, we want to see if any estimator can be accurate at least partially. In particular, we consider the naive estimator that operates on the assumption that samples are from the aggregated model $p_{\tilde{\mathcal{\tau}}, \tilde{q}}$. There is no reason that the naive estimates should reflect the parameters associated with the true model $p_{\mathcal{T}, q}$. Even in the slow mixing case, we want to see if any estimator can be accurate at least partially. In particular, we consider the naive estimator that operates on the assumption that samples are from the aggregated model $p_{\tilde{\tau}, \tilde{q}}$. Without our assumption on dependencies falling off, there is no a-priori reason that the naive estimates should reflect the parameters associated with the true model $p_{\mathcal{T}, q}$.

Definition 3. Given a sample sequence $Y_{1}^{n}$, let $\tilde{\mathcal{T}}=\mathcal{A}^{k_{n}}$ with $k_{n}=\alpha_{n} \log n$ for some function $\alpha_{n}=\mathcal{O}(1)$. For $\mathbf{s} \in \tilde{\mathcal{T}}$ let $\mathbf{Y}_{\mathbf{s}}$ be the sequence of symbols that follows the string $\mathbf{s}$. Hence, the length of $\mathbf{Y}_{\mathbf{s}}$ is

$$
N_{\mathbf{s}}=\sum_{i=1}^{n} \mathbb{1}\left\{\mathbf{c}_{\tilde{\mathcal{T}}}\left(Y_{-\infty}^{i-1}\right)=\mathbf{s}\right\}
$$


Therefore the number of $a^{\prime} s$ in $\mathbf{Y}_{\mathbf{s}}$ is

$$
n_{\mathbf{s} a}=\sum_{i=1}^{n} \mathbb{1}\left\{\mathbf{c}_{\tilde{\mathcal{T}}}\left(Y_{-\infty}^{i}\right)=\mathbf{s} a\right\} \text {. }
$$

Observer that $N_{\mathbf{s}}=\sum_{a \in \mathcal{A}} n_{\mathbf{s} a}$. The naive estimate of the aggregated parameters is

$$
\hat{q}(a \mid \mathbf{s})=\frac{n_{\mathbf{s} a}}{N_{\mathbf{s}}}
$$

Remark Note that $\mathbf{Y}_{\mathbf{s}}$ is i.i.d. only if $\mathbf{s} \in \mathcal{T}$, the set of states for the true model. In general, since we do not necessarily know if any of $n_{\mathbf{s} a}$ reflect the stationary frequencies, there is no obvious reason why $\hat{q}(a \mid \mathbf{s})$ shall reflect $\tilde{q}(a \mid \mathbf{s})$.

Since the process could be slow mixing, not all parameters are going to be accurate. Rather, there will be a set of good states in which we can do estimation properly.

Let $\delta_{j}=\sum_{i \geq j} d(i)$. Note that $\delta_{j} \rightarrow 0$ as $j \rightarrow \infty$ and that $-\delta_{j} \log \delta_{j} \rightarrow 0$ as $\delta_{j} \rightarrow 0$.

Definition 4. Given a sample sequence with size $n$ from $p_{\mathcal{T}, q}$, let

$\tilde{G}=\left\{\mathbf{w} \in \tilde{\mathcal{T}}: N_{\mathbf{w}} \geq \max \left\{n \delta_{k_{n}} \log \frac{1}{\delta_{k_{n}}},|\mathcal{A}|^{k_{n}+1} \log ^{2} n\right\}\right\}$.

Note that the set $\tilde{G}$ is obtained only using the sample and the known function $d$. We call the set $\tilde{G}$ "good" in an anticipatory fashion because we are going to prove concentration results for estimates attached to these states.

Secondly, since we include arbitrary slow mixing sources, there is no way to estimate all conditional probabilities with a sample of length $n$. Therefore, while the definition of $\tilde{G}$ above may not be the tightest, some notion of good states is unavoidable.

Remark Throughout this paper, we assume that we start with some past $Y_{-\infty}^{0}$, and we see $n$ samples $Y_{1}^{n}$ from $p_{\mathcal{T}, q}$. All confidence probabilities are conditional probabilities on $Y_{1}^{n}$ obtained from underlying unknown model $p_{\mathcal{T}, q}$, given past symbols $Y_{-\infty}^{0}$. The results in Sections X XII hold for all $Y_{-\infty}^{0}$ (not just with probability 1). In addition, if the history is not available, we can consider the first $k_{n}$ sample as the history and compute the empirical counts from the $n-k_{n}$ remaining samples. In other words, we can consider our length- $n$ sample as if we observe $Y_{-k_{n}+1}^{n-k_{n}}$ where $Y_{-k_{n}+1}^{0}$ can be thought as the needed history in computing the naive estimators. Since $k_{n}=\mathcal{O}(\log n)$ is negligible compared to $n$, in the rest we skip this complication in computing empirical counts for the sake of simplification.

\section{Estimate of Transition Probabilities}

Let $\tilde{\mathcal{T}}=\mathcal{A}^{k_{n}}$. Using samples from $p_{\mathcal{T}, q}$, we consider the estimation of parameters $\tilde{q}(\tilde{\mathcal{T}})$ of the aggregated model at depth $k_{n}$, and derive deviation bounds on the estimates in Theorem 2. However, before going to the proof of theorem, we want to make the following important remark.
Remark Observe that because we do not assume the source has mixed, the theorem below does not imply that the parameters are accurate for contexts shorter than $k_{n}$. We may therefore be able handle longer states' parameters (say a sequence of ten 0 s), without being able to infer those attached to their suffixes (say a sequence of five 0s).

This is perhaps counterintuitive at first glance. To see why this could happen, note that the result below shows what can be obtained without using anything about the mixing properties of the source-namely, it does not rely on empirical frequencies of various strings being close to their stationary probabilities. Therefore, results on longer strings do not automatically translate to results on shorter ones. Secondly, longer strings have attached conditional probabilities closer to the true conditional probabilities with which the source generates the data-therefore, there is less bias to counter.

This is contrary to most prior work which obtain bounds on transition probabilities subsequent to concentration of empirical counts of strings around their stationary probabilities. Additional information about the mixing of the source would further strengthen the following results.

Theorem 2. Let $Y_{1}^{n}$ be generated by an unknown model $p_{\mathcal{T}, q} \in \mathcal{M}_{d}$. Let $k_{n}=\alpha_{n} \log n$. Given any $Y_{-\infty}^{0}$, with probability under $p_{\mathcal{T}, q} \geq 1-\frac{1}{2^{|\mathcal{A}|^{k n+1} \log n}}$, for all $\mathbf{w} \in \tilde{\mathcal{T}}$ simultaneously

$$
D(\hat{q}(\cdot \mid \mathbf{w}) \| \tilde{q}(\cdot \mid \mathbf{w})) \leq \frac{2\left(|\mathcal{A}|^{k_{n}+1} \log n+n \delta_{k_{n}}\right)}{N_{\mathbf{w}}} .
$$

Proof As before, let $\delta_{k_{n}}=\sum_{i \geq k_{n}} d(i)$ and let $n$ be large enough that $\delta_{k_{n}} \leq \frac{1}{2}$. Note that Proposition 3 implies that for all sequences $y_{1}^{n} \in \mathcal{A}^{n}$ and all $Y_{-\infty}^{0}$

$$
\begin{aligned}
p_{\mathcal{T}, q}\left(y_{1}^{n} \mid Y_{-\infty}^{0}\right) & \leq \frac{1}{\left(1-\delta_{k_{n}}\right)^{n}} \prod_{\mathbf{w} \in \tilde{\mathcal{T}}} \prod_{a \in \mathcal{A}} \tilde{q}(a \mid \mathbf{w})^{n_{\mathbf{w} a}} \\
& \leq 4^{n \delta_{k_{n}}} \prod_{\mathbf{w} \in \tilde{\mathcal{T}}} \prod_{a \in \mathcal{A}} \tilde{q}(a \mid \mathbf{w})^{n_{\mathbf{w} a}},
\end{aligned}
$$

where $n_{\mathbf{w} a}$ was defined in Definition 3 and the second inequality is because $\left(\frac{1}{1-t}\right)^{n} \leq 4^{n t}$ whenever $0 \leq t \leq \frac{1}{2}$. Now, let $B_{n}$ be the set of all sequences that satisfy

$$
4^{n \delta_{k_{n}}} \prod_{\mathbf{w} \in \tilde{\mathcal{T}}} \prod_{a \in \mathcal{A}} \tilde{q}(a \mid \mathbf{w})^{n_{\mathbf{w} a}} \leq \frac{\prod_{\mathbf{w} \in \tilde{\mathcal{T}}} \prod_{a \in \mathcal{A}} \hat{q}(a \mid \mathbf{w})^{n_{\mathbf{w} a}}}{2^{2|\mathcal{A}|^{k_{n}+1} \log n}} .
$$

Using a depth- $k_{n}$ context tree weighting algorithm [21] we obtain a distribution $p_{c}$ satisfying 9

$$
p_{c}\left(y_{1}^{n} \mid Y_{-\infty}^{0}\right) \geq \frac{\prod_{\mathbf{w} \in \tilde{\mathcal{T}}} \prod_{a \in \mathcal{A}} \hat{q}(a \mid \mathbf{w})^{n_{\mathbf{w} a}}}{2^{|\mathcal{A}|^{k_{n}+1} \log n}} .
$$

Now, for all sequences $y_{1}^{n} \in B_{n}$, we have

$$
\begin{aligned}
p_{c}\left(y_{1}^{n} \mid Y_{-\infty}^{0}\right) & \geq \frac{\prod_{\mathbf{w} \in \tilde{\mathcal{T}}} \prod_{a \in \mathcal{A}} \hat{q}(a \mid \mathbf{w})^{n_{\mathbf{w} a}}}{2^{|\mathcal{A}|^{k_{n}+1} \log n}} \\
& \geq \frac{4^{n \delta_{k_{n}}} \prod_{\mathbf{w} \in \tilde{\mathcal{T}}} \prod_{a \in \mathcal{A}} \tilde{q}(a \mid \mathbf{w})^{n_{\mathbf{w} a}} 2^{2|\mathcal{A}|^{k_{n}+1} \log n}}{2^{|\mathcal{A}|^{k_{n}+1} \log n}} \\
& \geq p_{\mathcal{T}, q}\left(y_{1}^{n} \mid Y_{-\infty}^{0}\right) 2^{|\mathcal{A}|^{k_{n}+1} \log n}
\end{aligned}
$$

${ }^{9}$ While we use the context tree weighting algorithm, any worst case optimal universal compression algorithm would do for this theorem to follow. 
Thus, $B_{n}$ is the set of sequences $y_{1}^{n}$ such that $p_{c}$ assigns a much higher probability than $p_{\tau, q}$. Such a set $B_{n}$ can not have high probability under $p_{\mathcal{T}, q}$.

$$
\begin{aligned}
& p_{\mathcal{T}, q}\left(B_{n}\right)= \\
& p_{\mathcal{T}, q}\left\{y_{1}^{n}: p_{c}\left(y_{1}^{n} \mid Y_{-\infty}^{0}\right) \geq p_{\mathcal{T}, q}\left(y_{1}^{n} \mid Y_{-\infty}^{0}\right) 2^{|\mathcal{A}|^{k_{n}+1} \log n}\right\} \\
& \quad \leq \sum_{y_{1}^{n} \in B_{n}} p_{c}\left(y_{1}^{n} \mid Y_{-\infty}^{0}\right) 2^{-|\mathcal{A}|^{k_{n}+1} \log n} \\
& \quad \leq 2^{-|\mathcal{A}|^{k_{n}+1} \log n} .
\end{aligned}
$$

Therefore, with probability $\geq 1-2^{-|\mathcal{A}|^{k_{n}+1} \log n}$, (no matter $Y_{-\infty}^{0}$ ) we have

$$
\prod_{\mathbf{w} \in \tilde{\mathcal{T}}} \prod_{a \in \mathcal{A}} \tilde{q}(a \mid \mathbf{w})^{n_{\mathbf{w} a}} \geq \frac{\prod_{\mathbf{w} \in \tilde{\mathcal{T}}} \prod_{a \in \mathcal{A}} \hat{q}(a \mid \mathbf{w})^{n_{\mathbf{w} a}}}{2^{2|\mathcal{A}|^{k_{n}+1} \log n} 4^{n \delta_{k_{n}}}},
$$

which implies simultaneously for all $\mathbf{w} \in \tilde{\mathcal{T}}$

$$
\prod_{a \in \mathcal{A}} \tilde{q}(a \mid \mathbf{w})^{n_{\mathbf{w} a}} \geq \frac{\prod_{a \in \mathcal{A}} \hat{q}(a \mid \mathbf{w})^{n_{\mathbf{w} a}}}{2^{2|\mathcal{A}|^{k_{n}+1} \log n} 4^{n \delta_{k_{n}}}} .
$$

The above equation implies that $\tilde{q}$ and $\hat{q}$ are close distributions, since we can rearrange (and divide both sides by $N_{\mathbf{w}}$ ) to obtain:

$$
\begin{aligned}
\sum_{a \in \mathcal{A}} \frac{n_{\mathbf{w} a}}{N_{\mathbf{w}}} \log \frac{\hat{q}(a \mid \mathbf{w})}{\tilde{q}(a \mid \mathbf{w})} & =D(\hat{q}(\cdot \mid \mathbf{w}) \| \tilde{q}(\cdot \mid \mathbf{w})) \\
& \leq \frac{2\left(|\mathcal{A}|^{k_{n}+1} \log n+n \delta_{k_{n}}\right)}{N_{\mathbf{w}}},
\end{aligned}
$$

where the first equality follows by writing out the value of the naive estimate, $\hat{q}(a \mid \mathbf{w})=n_{\mathbf{w} a} / N_{\mathbf{w}}$.

Corollary 3. Let $Y_{1}^{n}$ be generated by an unknown model $p_{\mathcal{T}, q} \in \mathcal{M}_{d}$. Let $k_{n}=\alpha_{n} \log n$. Given any $Y_{-\infty}^{0}$, with probability under $p_{\mathcal{T}, q} \geq 1-\frac{1}{2^{|\mathcal{A}|^{k_{n}+1} \log n}}$, for all $\mathbf{w} \in \tilde{\mathcal{T}}$ simultaneously

$$
\|\tilde{q}(\cdot \mid \mathbf{w})-\hat{q}(\cdot \mid \mathbf{w})\|_{1} \leq 2 \sqrt{\frac{(\ln 2)\left(|\mathcal{A}|^{k_{n}+1} \log n+n \delta_{k_{n}}\right)}{N_{\mathbf{w}}}} .
$$

Proof The proof follows immediately from Theorem 2 and Pinsker's inequality (see e.g., [43])

$$
D(\hat{q}(\cdot \mid \mathbf{w}) \| \tilde{q}(\cdot \mid \mathbf{w})) \geq \frac{1}{2 \ln 2}\|\tilde{q}(\cdot \mid \mathbf{w})-\hat{q}(\cdot \mid \mathbf{w})\|_{1}^{2} .
$$

Remark We emphasize that the above results depend on $Y_{-\infty}^{0}$ hold for all history $Y_{-\infty}^{0}$.

Remark For all $\mathbf{w} \in \tilde{\mathcal{T}}$ with $|\mathcal{A}|=2$, by Corollary 3 , we have

$$
\begin{aligned}
& \|\tilde{q}(. \mid \mathbf{w})-\hat{q}(. \mid \mathbf{w})\|_{1} \leq \\
& \begin{cases}2 \sqrt{\frac{(2 \ln 2) 2^{k_{n}+1} \log n}{N_{\mathbf{w}}}} & \text { if } k_{n} \geq \log \left(\frac{n \delta_{k_{n}}}{2 \log n}\right), \\
2 \sqrt{\frac{(2 \ln 2) n \delta_{k_{n}}}{N_{\mathbf{w}}}} & \text { otherwise. }\end{cases}
\end{aligned}
$$

If $d(i)=\gamma^{i}$ for some $0<\gamma<\frac{1}{2}$, then $\delta_{k_{n}}=\frac{\gamma^{k_{n}}}{1-\gamma}$ and by choosing $k_{n}=\gamma \log n$, the accuracy of above estimation is

$$
\Theta\left(\sqrt{\frac{n^{1+\gamma \log \gamma}}{N_{\mathbf{w}}}}\right) .
$$

Therefore if $N_{\mathbf{w}}=\frac{n}{\log n}$, then the accuracy of the estimation will be $\Theta\left(\sqrt{n^{\gamma \log \gamma \log n}}\right)$.

If $\frac{1}{2}<\gamma<1$, then the accuracy will be

$$
\Theta\left(\sqrt{\frac{n^{\gamma} \log n}{N_{\mathbf{w}}}}\right) .
$$

Therefore if $N_{\mathbf{w}}=\frac{n}{\log n}$, then the accuracy of the estimation will be $\Theta\left(n^{\frac{\gamma-1}{2}} \log n\right)$.

If $d(i)=\frac{1}{i^{r}}$ for some $r>2$, then $\delta_{k_{n}} \approx k_{n}{ }^{1-r}$ and by choosing $k_{n}=\frac{1}{r} \log n-\log \log n$, the accuracy will be

$$
\Theta\left(\sqrt{\frac{n}{k_{n}^{r-1} N_{\mathbf{w}}}}\right) .
$$

Therefore, if $N_{\mathbf{w}}=\frac{n}{\log n}$, then the accuracy of the estimation will be $\Theta\left(\sqrt{\frac{1}{(\log n)^{r-2}}}\right)$.

Good states $\tilde{G}$ in Definition (4) identify all strings $\mathbf{w}$ in the sample whose transition probabilities are accurate to at least $\frac{1}{\sqrt{\log n}}$. It is quite possible that for all $\mathbf{w} \in \tilde{G}$, the accuracy is significantly better.

When the dependencies among strings die down exponentially, we can strengthen Theorem 2 with a more careful calculations to get a stronger convergence rate polynomial in $n$.

Theorem 4. Suppose $d(i)=\gamma^{i}$ for some $0<\gamma<1$. Let $\zeta$ be a nonnegative constant. Let $k_{n}=\frac{\log n}{\log \frac{|\mathcal{A}|}{\gamma}}$. In analogy with $\tilde{G}$, we define

$$
\tilde{F} \triangleq\left\{\mathbf{w} \in \tilde{\mathcal{T}}: N_{\mathbf{w}} \geq n^{\zeta+\frac{\log |\mathcal{A}|}{\log |\mathcal{A}|-\log \gamma}}\right\} .
$$

Then, conditioned on any past $Y_{-\infty}^{0}$, with probability under $p_{\mathcal{T}, q}$ greater than $1-2^{-|\mathcal{A}|^{k_{n}+1} \log n}$ simultaneously for all $\mathbf{w} \in \tilde{F}$

$$
\|\tilde{q}(. \mid \mathbf{w})-\hat{q}(. \mid \mathbf{w})\|_{1} \leq 2 \sqrt{\frac{\ln 2 \cdot((1-\gamma)|\mathcal{A}| \log n+1)}{(1-\gamma) n^{\zeta}}} .
$$

Proof The proof of Theorem 4 is similar to Theorem 2 but involves more careful but elementary algebra specific to the exponential decay case using the value of $k_{n}$ and $\tilde{F}$ noted in the statement. Note that $\zeta<\frac{\log \frac{1}{\gamma}}{\log \frac{|\mathcal{A}|}{\gamma}}$ for the Theorem not to be vacuously true.

Remark According to definition of good states in Theorem 4 and the fact that $\tilde{\mathcal{T}}=\mathcal{A}^{k_{n}}$, we obtain

$$
|\tilde{F}| \leq n^{-\frac{\log \gamma}{\log |\mathcal{A}|-\log \gamma}},|\tilde{\mathcal{T}}|=n^{\frac{\log |\mathcal{A}|}{\log |\mathcal{A}|-\log \gamma}}
$$

implying that if $\gamma \leq 1 /|\mathcal{A}|$, all states of $\tilde{\mathcal{T}}$ can potentially be good.

Remark The rate of convergence in Theorem 2 is the minimum that hold for all strings of length $k_{n}$ simultaneously, not just good strings- and strings that appear more often will 
have stronger bounds automatically. Specifically, strings that appear $n^{\beta}$ times for any $\beta>0$ have convergence rates that are polynomial in $n$ for the exponential decay case. We emphasize that our results are not too off because even in i.i.d. case, the Chernoff bounds do not provide much stronger relative to the bounds we obtain in the exponential decay case.

\section{Xi. Estimation Along a Sequence of Stopping TIMES}

As we saw in the prior section, the aggregated parameters $\tilde{q}(\cdot \mid \mathbf{w})$ associated with any good state $\mathbf{w} \in \tilde{G}$ can be approximated from the sample. From Example 4. we know that the stationary probabilities may be a very sensitive function of the parameters associated with states. How do we tell, therefore, if the few conditional probabilities we estimated can say anything at all about stationary probabilities?

We explore these questions in the next two sections. In order to interpret the counts of various strings $\mathbf{w}$ in the sample, we first study the process $\left\{Y_{i}\right\}_{i \geq 1}$ from $p_{\mathcal{T}, q}$ restricted to states in $\tilde{G}$ in this section. Some of the observations regarding stopping times in this section are well known, see for example [47].

In Section XII, we use these observations with a coupling argument to derive Theorem 7 that provides deviation bounds on the counts of strings. Note that any deviation result must also keep in mind the fundamental difficulty that the probability under the stationary distribution of all strings in the observed sample could be arbitrarily small, no matter the size of the sample, as illustrated in Example 2.

\section{A. Restriction of $p_{\mathcal{T}, q}$ to $\tilde{G}$}

To find deviation bounds for stationary distribution of good states, we construct a new process $\left\{Z_{m}\right\}_{m \geq 1}, Z_{m} \in \mathcal{T}$ from the process $\left\{Y_{i}\right\}_{i \geq 1}$. At the outset, note that $Z_{m} \in \mathcal{T}$, where $\mathcal{T}$ is unknown. We use this process $\left\{Z_{m}\right\}_{m \geq 1}$ as an analytical tool, and we will not need to actually observe it. We will need to know if the process is aperiodic, but that can be resolved by only looking at $\tilde{G}$.

If $Y_{i_{m}}$ is the $(m+1)^{t h}$ symbol in the sequence $\left\{Y_{i}\right\}_{i \geq 1}$ such that $\mathbf{c}_{\tilde{\mathcal{T}}}\left(Y_{-\infty}^{i_{m}}\right) \in \tilde{G}$, then $Z_{m}=\mathbf{c}_{\mathcal{T}}\left(Y_{-\infty}^{i_{m}}\right)$. The strong Markov property [48] allows us to characterize $\left\{Z_{m}\right\}_{m \geq 1}$ as a Markov process with transitions that are lower bounded by those transitions of the process $\left\{Y_{i}\right\}_{i \geq 1}$ that can be well estimated by the Theorems above. More specifically, let

$$
T_{0}=\min \left\{j \geq 0: \mathbf{c}_{\tilde{\mathcal{T}}}\left(Y_{-\infty}^{j}\right) \in \tilde{G}\right\},
$$

and let $Z_{0}=\mathbf{c}_{\mathcal{T}}\left(Y_{-\infty}^{T_{0}}\right)$. For all $m \geq 1, T_{m}$ is the $(m+1)^{\prime}$ th occurrence of a good state in the sequence $\left\{Y_{i}\right\}_{i \geq 1}$, namely

$$
T_{m}=\min \left\{j>T_{m-1}: \mathbf{c}_{\tilde{\mathcal{T}}}\left(Y_{-\infty}^{j}\right) \in \tilde{G}\right\},
$$

and $Z_{m}=\mathbf{c}_{\mathcal{T}}\left(Y_{-\infty}^{T_{m}}\right)$. Note that $T_{m}$ is a stopping time [5], and therefore $\left\{Z_{m}\right\}_{m>1}$ is a Markov chain by itself ${ }^{10}$ Let $\tilde{B}=$ $\{\mathbf{s} \in \tilde{\mathcal{T}}: \mathbf{s} \notin \tilde{G}\}$. The transitions between states $\mathbf{w}, \mathbf{w}^{\prime} \in \tilde{G}$

\footnotetext{
${ }^{10}$ Note that strong Markov property implies that the Markov property holds at stopping times.
}

are then the minimal, non-negative solution of the following set of equations in $\left\{Q(\mathbf{w} \mid \mathbf{s}): \mathbf{s} \in \mathcal{A}^{k_{n}}, \mathbf{w} \in \tilde{G}\right\}$

$$
\begin{aligned}
Q\left(\mathbf{w} \mid \mathbf{w}^{\prime}\right) & =p_{\mathcal{T}, q}\left(\mathbf{c}_{\tilde{\mathcal{T}}}\left(Y_{-\infty}^{1}\right)=\mathbf{w} \mid \mathbf{c}_{\tilde{\mathcal{T}}}\left(Y_{-\infty}^{0}\right)=\mathbf{w}^{\prime}\right) \\
& +\sum_{\mathbf{s} \in \tilde{B}} Q(\mathbf{w} \mid \mathbf{s}) p_{\mathcal{\mathcal { T } , q}}\left(\mathbf{c}_{\tilde{\mathcal{T}}}\left(Y_{-\infty}^{1}\right)=\mathbf{s} \mid \mathbf{c}_{\tilde{\mathcal{T}}}\left(Y_{-\infty}^{0}\right)=\mathbf{w}^{\prime}\right) .
\end{aligned}
$$

An important point to note here is that if $\mathbf{w}$ and $\mathbf{w}^{\prime}$ are good states,

$$
Q\left(\mathbf{w} \mid \mathbf{w}^{\prime}\right) \geq p_{\mathcal{T}, q}\left(\mathbf{c}_{\tilde{\mathcal{T}}}\left(Y_{-\infty}^{1}\right)=\mathbf{w} \mid \mathbf{c}_{\tilde{\mathcal{T}}}\left(Y_{-\infty}^{0}\right)=\mathbf{w}^{\prime}\right),
$$

and the lower bound above can be well estimated from the sample as shown in Theorem 2 .

Definition 5. We will call $\left\{Z_{m}\right\}_{m \geq 1}$, the restriction of $p_{\mathcal{T}, q}$ to $\tilde{G}$.

\section{B. Properties of $\left\{Z_{m}\right\}_{m \geq 1}$}

Property 1. A few properties about $\left\{Z_{m}\right\}_{m \geq 1}$ are in order. $\left\{Z_{m}\right\}_{m \geq 1}$ is constructed from an irreducible process $\left\{Y_{i}\right\}_{i \geq 1}$, thus $\left\{Z_{m}\right\}_{m \geq 1}$ is irreducible as well. Since $\left\{Y_{i}\right\}_{i \geq 1}$ is positive recurrent, so is $\left\{Z_{m}\right\}_{m \geq 1}$. But despite $\left\{Y_{i}\right\}_{i \geq 1}$ being aperiodic, $\left\{Z_{m}\right\}_{m \geq 1}$ could be periodic as in the Example below. But periodicity of $\left\{Z_{m}\right\}_{m \geq 1}$ can be determined by $\tilde{G}$ alone (because $\mathcal{T}$, while unknown, is a full, finite $\mathcal{A}$-ary tree).

Example 8. Let $\left\{Y_{i}\right\}_{i \geq 1}$ be a process generated by context tree model $p_{\mathcal{T}, q}$ with $\mathcal{T}=\{11,01,10,00\}$ and $q(1 \mid 11)=$ $\frac{1}{2}, q(1 \mid 01)=\epsilon, q(1 \mid 10)=1-\epsilon, q(1 \mid 00)=\frac{1}{2}$. If $\epsilon>0$, then $p_{\mathcal{T}, q}$ represents a stationary aperiodic Markov process. If $\left\{Z_{m}\right\}_{m \geq 1}$ be the restriction of process $\left\{Y_{i}\right\}_{i \geq 1}$ to $\tilde{G}=$ $\{01,10\}$, the restricted process will be periodic with period 2 .

Property 2. Suppose $\left\{Z_{m}\right\}_{m>1}$ is aperiodic. Let $\pi_{Y}$ and $\pi_{Z}$ denote the stationary distribution of the processes $\left\{Y_{i}\right\}_{i \geq 1}$ and $\left\{Z_{m}\right\}_{m \geq 1}$, respectively, with $n$ samples of a sequence $\left\{Y_{i}\right\}_{i \geq 1}$ yielding $m_{n}$ samples of $\left\{Z_{m}\right\}_{m \geq 1}$. Similarly, let $\pi_{Z}(\mathbf{w})$ denote the stationary probability of the event that $\mathbf{w}$ is a suffix of samples in the process $\left\{Z_{m}\right\}_{m \geq 1}$. Then for all $\mathbf{w}, \mathbf{w}^{\prime} \in \tilde{G}$ (note that $\pi_{Y}\left(\mathbf{w}^{\prime}\right)>0$ )

$$
\begin{aligned}
& \frac{\pi_{Y}(\mathbf{w})}{\pi_{Y}\left(\mathbf{w}^{\prime}\right)} \stackrel{w p}{=} \frac{\lim _{n \rightarrow \infty} \sum_{i=1}^{n} \frac{\mathbb{1}\left(\mathbf{c}_{\tilde{\mathcal{T}}}\left(Y_{-\infty}^{i}\right)=\mathbf{w}\right)}{n}}{\lim _{n \rightarrow \infty} \sum_{i=1}^{n} \frac{\mathbb{1}\left(\mathbf{c}_{\tilde{\mathcal{T}}}\left(Y_{-\infty}^{i}\right)=\mathbf{w}^{\prime}\right)}{n}} \\
&=\frac{\lim _{n \rightarrow \infty} \sum_{j=1}^{m_{n}} \frac{\mathbb{1}\left(\mathbf{w} \preceq Z_{j}\right)}{m_{n}}}{\lim _{m_{n} \rightarrow \infty} \sum_{j=1}^{m_{n}} \frac{\mathbb{1}\left(\mathbf{w}^{\prime} \preceq Z_{j}\right)}{m_{n}}} \\
& \stackrel{w p 1}{=} \frac{\pi_{Z}(\mathbf{w})}{\pi_{Z}\left(\mathbf{w}^{\prime}\right)} .
\end{aligned}
$$

\section{Estimate Of Stationary Probabilities}

Thus far, we have identified a set $\tilde{G} \subseteq \mathcal{A}^{k_{n}}$ of good strings using $n$ observations from $p_{\mathcal{T}, q}$. For strings $\mathbf{w} \in \tilde{G}$, we have been able to estimate approximately the conditional distributions, conditioned on past strings $\mathbf{w}$-namely $P(Y \mid \mathbf{w})$, or equivalently the aggregated parameters $\tilde{q}(\cdot \mid \mathbf{w})$. As mentioned 
before, it is not clear if this information we have obtained from the sample will allow us to say anything at all about the stationary probabilities of strings $\mathbf{w} \in \tilde{G}$. This section develops on this question, and shows how to interpret naive counts of $\mathbf{w} \in \tilde{G}$ in the sample.

\section{A. Outline}

Our main result in this section is Theorem 7 a concentration result on the counts $N_{\mathbf{w}}$. This follows essentially as a martingale convergence bound. Consider the process restricted to the good states $\tilde{G}$, namely, $\left\{Z_{i}\right\}_{i \geq 0}$. In Definition 6 , we consider the natural Doob martingale $V_{m} \stackrel{\text { def }}{=} \mathbb{E}\left[N_{\mathbf{w}} \mid Z_{0}, Z_{1}, \ldots, Z_{m}\right]$. In Subsection XII-C, we give a bound on the differences $\left|V_{m}-V_{m+1}\right|$ using a variation of the coupling argument. In Subsection XII-D we construct a specific coupling that is analyzed in XII-F to provide a bound on the martingale differences. Theorem 7 then follows from Azuma's inequality.

\section{B. Preliminaries}

For any (good) state $\mathbf{w}$, let $G_{\mathrm{w}} \subset \mathcal{A}$ be the set of letters that take $\mathbf{w}$ to another good state,

$$
G_{\mathbf{w}}=\left\{a \in \mathcal{A}: \mathbf{c}_{\tilde{\mathcal{T}}}(\mathbf{w} a) \in \tilde{G}\right\} .
$$

Our confidence in the empirical counts of good states matching their (aggregated) stationary probabilities follows from a coupling argument, and depends on the following parameter

$$
\eta_{\tilde{G}}=\min _{\mathbf{u}, \mathbf{v} \in \tilde{G}} \sum_{a \in G_{\mathbf{u}} \cap G_{\mathbf{v}}} \min \{\tilde{q}(a \mid \mathbf{u}), \tilde{q}(a \mid \mathbf{v})\},
$$

where $\tilde{q}(a \mid \mathbf{u})$ is as defined before in $(5)$ for aggregated model parameters. The parameter $\eta_{\tilde{G}}$ is closely related to the notion of Dobrushin's ergodicity coefficient of Markov processes (see e.g., [26]). The parameter $\eta_{\tilde{G}}$ is different from standard Dobrushin's ergodicity coefficient in two aspects. First, notice that in $(8), \mathbf{u}$ and $\mathbf{v}$ might not be the states of $p_{\mathcal{T}, q}$. Second, the summation index runs over those alphabets which take both states $\mathbf{u}$ and $\mathbf{v}$ to any other good state in $\tilde{G}$. Note that for any state $\mathbf{s} \in \mathcal{T}$ of the original process $p_{\mathcal{T}, q}$, if $\mathbf{u} \preceq \mathbf{s}$, using Proposition 3

$$
\tilde{q}(a \mid \mathbf{u}) \leq \frac{q(a \mid \mathbf{s})}{1-\delta_{|\mathbf{u}|}}
$$

Remark Recall that the deviation bounds in Theorems 2 and 4 hold simultaneously for all contexts in $\tilde{G}$. The above definition only depends on parameters associated with $\tilde{G}$. Hence we can estimate $\eta_{\tilde{G}}$ from the sample with the same confidence and (twice the) error given in those Theorems.

The counts of various $\mathbf{w} \in \tilde{G}$ then concentrates as shown in the Theorem 7 and how good the concentration is can be estimated as a function of $\eta_{\tilde{G}}$ (and $\delta_{k_{n}}$ ) and the total number of times states in $\tilde{G}$ occur in the sample. Now $\tilde{G}$ as well as $\eta_{\tilde{G}}$ are well estimated from the sample-thus we can look at the data to interpret the empirical counts of various substrings of the data.

Let

$$
\Delta_{j}=\sum_{i \geq j} \delta_{i}
$$

For the following results, we require $\left\{\delta_{i}\right\}_{i \geq 1}$ to be summable. Thus, $\Delta_{j}$ is finite for all $j$ and decreases to 0 as $j$ increases. If $d(i) \sim \gamma^{i}$, then $\Delta_{j}$ also diminishes as $\gamma^{j}$. But if $d(i) \sim$ $\frac{1}{i^{r}}$ diminishes polynomially, then $\Delta_{j}$ diminishes as $1 / j^{r-2}$. If $d(i)=1 / i^{2+\eta}$ for any $\eta>0$, we therefore satisfy the summability of $\left\{\delta_{i}\right\}_{i \geq 1}$. However, $d(i)$ can also diminish as $1 /\left(i^{2}\right.$ poly $\left.(\log i)\right)$ for appropriate polynomials of $\log i$ for the counts of good states to converge. In what follows, we assume that $\delta_{i} \leq \frac{1}{i}$.

Definition 6. Let $\tilde{G}$ be the set of good states from Definition 4 Let $\tilde{n}$ be total count of all good states in the sample and $\ell_{n}$ denote the smallest integer such that $\Delta_{\ell_{n}} \leq \frac{1}{n}$. To analyze the naive counts of $\mathbf{w} \in \tilde{G}$, we define

$$
V_{m} \stackrel{\text { def }}{=} \mathbb{E}\left[N_{\mathbf{w}} \mid Z_{0}, Z_{1}, \ldots, Z_{m}\right],
$$

where $N_{\mathbf{w}}$ is as in Definition 3 .

Observe that $\left\{V_{m}\right\}_{m=0}^{\tilde{n}}$ is a Doob martingale. Note that

$$
V_{0}=\mathbb{E}\left[N_{\mathbf{w}} \mid Z_{0}\right] \text { and } V_{\tilde{n}}=N_{\mathbf{w}} \text {. }
$$

Once again, note that we do not have to observe the restriction process $\left\{Z_{m}\right\}_{m \geq 1}$ at any point, nor do we have to observe the martingale $V$ except for noting $V_{\tilde{n}}=N_{\mathbf{w}}$.

Remark To prove Theorem 7, we first bound the differences $\left|V_{m}-V_{m-1}\right|$ of the martingale using a coupling argument in Lemma 5 Since the memory of the process $p_{\mathcal{T}, q}$ could be large, our coupled chains may not actually coalesce in the usual sense. But they get "close enough" that the chance they diverge again within $n$ samples is less than $1 / n$. Once we bound the differences in the martingale $\left\{V_{m}\right\}_{m=0}^{\tilde{n}}$, Theorem 7 follows as an easy application of Azuma's inequality.

\section{The Coupling Argument}

Since for all $m \geq 1$

$$
\begin{array}{r}
\left|V_{m}-V_{m-1}\right|=\left|\mathbb{E}\left[N_{\mathbf{w}} \mid Z_{0}, \ldots, Z_{m}\right]-\mathbb{E}\left[N_{\mathbf{w}} \mid Z_{0}, \ldots, Z_{m-1}\right]\right| \\
\quad \leq \max _{Z_{m}^{\prime}, Z_{m}^{\prime \prime}}\left|\mathbb{E}\left[N_{\mathbf{w}} \mid Z_{0}, \ldots, Z_{m}^{\prime}\right]-\mathbb{E}\left[N_{\mathbf{w}} \mid Z_{0}, \ldots, Z_{m}^{\prime \prime}\right]\right|
\end{array}
$$

we bound the maximum change in $N_{\mathbf{w}}$ if the $m$ th good state was changed into another (good) state.

Suppose there are sequences $\left\{Z_{j}^{\prime}\right\}_{j=m}^{n}$ (starting from state $Z_{m}^{\prime}$ ) and $\left\{Z_{j}^{\prime \prime}\right\}_{j=m}^{n}$ (starting from state $Z_{m}^{\prime \prime}$ ), both faithful copies of the restriction of $p_{\mathcal{T}, q}$ to $\tilde{G}$ but coupled with a joint distribution $\mu$ to be described below. From the coupling argument of Section VI-B, we have for $\mathbf{w} \in \tilde{G}$ (hence $\left.|\mathbf{w}|=k_{n}\right)$ for all $\mu$

$$
\begin{aligned}
&\left|\mathbb{E}\left[N_{\mathbf{w}} \mid Z_{0}, \ldots, Z_{m}^{\prime}\right]-\mathbb{E}\left[N_{\mathbf{w}} \mid Z_{0}, \ldots, Z_{m}^{\prime \prime}\right]\right| \\
& \leq \sum_{j=m+1}^{n} \mu\left(Z_{j}^{\prime} \not ॅ Z_{j}^{\prime \prime}\right),
\end{aligned}
$$

where we use

$$
Z_{j}^{\prime} \stackrel{k_{n}}{\approx} Z_{j}^{\prime \prime} \text { for } \mathbf{c}_{\mathcal{A}^{k_{n}}}\left(Z_{j}^{\prime}\right)=\mathbf{c}_{\mathcal{A}^{k_{n}}}\left(Z_{j}^{\prime \prime}\right) .
$$

We will bound the right side of (10) above using properties of $\mu$ that we describe. Note that the summation goes up to $n$ since no matter what $Z_{m}^{\prime}$ and $Z_{m}^{\prime \prime}$ are, a length- $n$ sample 
can have at most $n$ good states on the right side of (10). The coupling technique is a convenient "thought experiment" that culminates in Theorem 7 giving a deviation bound on the naive counts of states. We do not actually have to generate the two chains as part of the estimation algorithm, nor do we need to observe the martingale $V$, except for noting $V_{\tilde{n}}=N_{\mathbf{w}}$.

In Subsection XII-D below, we describe $\mu$, highlight some properties of the described coupling in Subsection XII-E, and use the said properties to obtain a bound on the martingale difference in Subsection XII-F

\section{Description of Coupling $\mu$}

Suppose we have $Z_{j}^{\prime}$ and $Z_{j}^{\prime \prime}$. This subsection describes how to obtain next sample $Z_{j+1}^{\prime}$ and $Z_{j+1}^{\prime \prime}$ of the two coupled chains, namely how to sample from

$$
\mu\left(Z_{j+1}^{\prime}, Z_{j+1}^{\prime \prime} \mid Z_{j}^{\prime}, Z_{j}^{\prime \prime}\right) .
$$

Recall from section VI-B that individually taken, both $Z_{j+1}^{\prime}$ and $Z_{j+1}^{\prime \prime}$ are faithful evolutions of the restriction of $p_{\mathcal{T}, q}$ to $\tilde{G}$. However, given $Z_{j}^{\prime}$ and $Z_{j}^{\prime \prime}, Z_{j+1}^{\prime}$ and $Z_{j+1}^{\prime \prime}$ are not necessarily independent.

To obtain $Z_{j+1}^{\prime}$ and $Z_{j+1}^{\prime \prime}$, starting from states $Z_{j}^{\prime}$ and $Z_{j}^{\prime \prime}$ we run copies $\left\{Y_{j i}^{\prime}\right\}_{i \geq 1}$ and $\left\{Y_{j i}^{\prime \prime}\right\}_{i \geq 1}{ }^{11}$ of coupled chains individually faithful to $p_{\mathcal{T}, q}$. If $l$ is the smallest number such that

$$
\mathbf{c}_{\tilde{\mathcal{T}}}\left(Z_{j}^{\prime} Y_{j 1}^{\prime} \cdots Y_{j l}^{\prime}\right) \in \tilde{G}
$$

then

$$
Z_{j+1}^{\prime}=\mathbf{c}_{\mathcal{T}}\left(Z_{j}^{\prime} Y_{j 1}^{\prime} \cdots Y_{j l}^{\prime}\right) .
$$

Similarly for $Z_{j+1}^{\prime \prime}$.

1) Sampling from the joint distribution $\mu\left(Y_{j 1}^{\prime}, Y_{j 1}^{\prime \prime} \mid Z_{j}^{\prime}, Z_{j}^{\prime \prime}\right)$ :

While the following description appears verbose, Fig. 7. (b) represents the description pictorially. Specifically, the chains $\left\{Y_{j i}^{\prime}\right\}_{i \geq 1}$ and $\left\{Y_{j i}^{\prime \prime}\right\}_{i \geq 1}$ are coupled as follows. We generate a number $U_{j 1}$ uniformly distributed in $[0,1]$. Given $\left(Z_{j}^{\prime}\right.$ and $\left.Z_{j}^{\prime \prime}\right)$ with suffixes $\mathbf{u}$ and $\mathbf{v}$ respectively in $\tilde{G}$, we let $G_{\mathbf{u}} \in \mathcal{A}$ (and $G_{\mathrm{v}}$ similarly) be the set of symbols in $\mathcal{A}$ defined as in (7). We split the interval from 0 to 1 as follows: for all $a \in \mathcal{A}$, we assign intervals $r(a)$ of length $\min \{q(a \mid \mathbf{u}), q(a \mid \mathbf{v})\}$, in the following order: we first stack the above intervals corresponding to $a \in G_{\mathbf{u}} \cap G_{\mathbf{v}}$ (in any order) starting from 0 , and then we put in the intervals corresponding to all other symbols. Now let,

$$
\left(Y_{j 1}^{\prime}, Y_{j 1}^{\prime \prime}\right)=(a, a) \text { if } U_{j 1} \in r(a) .
$$

Let

$$
C(\mathcal{A})=\sum_{a^{\prime} \in \mathcal{A}} r\left(a^{\prime}\right)=\sum_{a^{\prime} \in \mathcal{A}} \min \left\{q\left(a^{\prime} \mid Z_{j}^{\prime}\right), q\left(a^{\prime} \mid Z_{j}^{\prime \prime}\right)\right\},
$$

be the part of the interval is already filled up. Thus if $U_{j 1}<C(\mathcal{A})$, equivalently with probability $C(\mathcal{A})$, the two chains output the same symbol. We use the rest of the interval

\footnotetext{
${ }^{11}$ Note that $\left\{Y_{j i}^{\prime}\right\}_{i \geq 1}$ and $\left\{Y_{j i}^{\prime \prime}\right\}_{i \geq 1}$ are sequences of symbols from $\mathcal{A}$, generated according to transitions defined by $p_{\mathcal{T}, q}$.
}

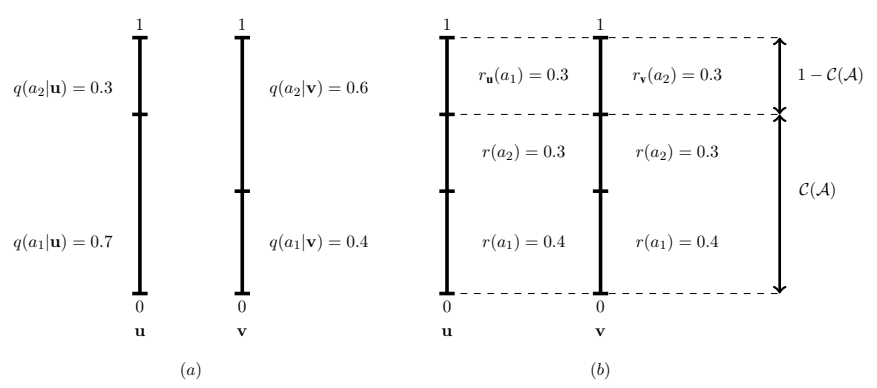

Fig. 7. (a) The conditional probabilities with which $Y_{j 1}^{\prime}$ and $Y_{j 1}^{\prime \prime}$ have to be chosen respectively are $q(\cdot \mid \mathbf{u})$ and $q(\cdot \mid \mathbf{v})$. The line on the left determines the choice of $Y_{j 1}^{\prime}$ and the one on the right the choice of $Y_{j 1}^{\prime \prime}$. For example, if $U_{j 1}$ is chosen uniformly in [0,1], the probability of choosing $Y_{j 1}^{\prime}=a_{1}$ is $q\left(a_{1} \mid \mathbf{u}\right)$. Instead of choosing $Y_{j 1}^{\prime}$ and $Y_{j 1}^{\prime \prime}$ independently, we will reorganize the intervals in the lines so as to encourage $Y_{j 1}^{\prime}=Y_{j 1}^{\prime \prime}$. (b) Reorganizing the interval $[0,1]$ according to the described construction. Here $r\left(a_{1}\right)=$ $\min \left\{q\left(a_{1} \mid \mathbf{u}\right), q\left(a_{1} \mid \mathbf{v}\right)\right\}$ and similarly for $r\left(a_{2}\right)$. If $U_{j 1}$ falls in the interval corresponding to $r\left(a_{1}\right)$, then $\left(Y_{j 1}^{\prime}, Y_{j 1}^{\prime \prime}\right)=\left(a_{1}, a_{1}\right)$. If $U_{j 1}>C(\mathcal{A})$ in this example, then $\left(Y_{j 1}^{\prime}, Y_{j 1}^{\prime \prime}\right)=\left(a_{1}, a_{2}\right)$. When $U_{j 1}$ is chosen uniformly in $[0,1]$, the probability $Y_{j 1}^{\prime}$ outputs any symbol is the same as in the picture on the left, similarly for $Y_{j 2}^{\prime}$.

$[C(A), 1]$ in any valid way to satisfy the fact that $Y_{j 1}^{\prime}$ is distributed as $p_{\mathcal{T}, q}\left(\cdot \mid Z_{j}^{\prime}\right)$ and $Y_{j 1}^{\prime \prime}$ is distributed as $p_{\mathcal{T}, q}\left(\cdot \mid Z_{j}^{\prime \prime}\right)$. For one standard approach, for all $a$ assign

$$
r_{\mathbf{u}}(a)=(q(a \mid \mathbf{u})-q(a \mid \mathbf{v}))^{+}=\max \{q(a \mid \mathbf{u})-q(a \mid \mathbf{v}), 0\}
$$

and similarly $r_{\mathbf{v}}(a)$. Note that only one of $r_{\mathbf{u}}(a)$ and $r_{\mathbf{v}}(a)$ can be strictly positive and that for all $a, r(a)+r_{\mathbf{u}}(a)=q(a \mid \mathbf{u})$ while $r(a)+r_{\mathbf{v}}(a)=q(a \mid \mathbf{v})$. Therefore,

$$
\sum_{a^{\prime} \in \mathcal{A}} r_{\mathbf{u}}\left(a^{\prime}\right)=\sum_{a^{\prime} \in \mathcal{A}} r_{\mathbf{v}}\left(a^{\prime}\right)=1-C(\mathcal{A}) .
$$

An example of such construction for binary alphabet is illustrated in Fig. 7 in which we have assumed $G_{\mathbf{u}} \cap G_{\mathbf{v}}=\left\{a_{1}\right\}$. We will keep two copies of the interval $[C(\mathcal{A}), 1]$, and if $U_{j 1}>C(\mathcal{A})$ we output $\left(Y_{j 1}^{\prime}, Y_{j 1}^{\prime \prime}\right)$ based on where $U_{j 1}$ falls in both copies. We will stack the first copy of $[C(\mathcal{A}), 1]$ with intervals of length $r_{\mathbf{u}}(a)$ for all $a$ and the second copy of $[C(\mathcal{A}), 1]$ with intervals length $r_{\mathbf{v}}(a)$ for all $a$. We say $U_{j 1} \in\left(r_{\mathbf{u}}(a), r_{\mathbf{v}}\left(a^{\prime}\right)\right)$ if $U_{j 1} \in r_{\mathbf{u}}(a)$ in the first copy and $U_{j 1} \in r_{\mathbf{v}}\left(a^{\prime}\right)$ in the second copy,

$$
\left(Y_{j 1}^{\prime}, Y_{j 1}^{\prime \prime}\right)=\left(a, a^{\prime}\right) \text { if } U_{j 1} \in\left(r_{\mathbf{u}}(a), r_{\mathbf{v}}\left(a^{\prime}\right)\right) .
$$

Note in particular that

$$
\mu\left(Y_{j 1}^{\prime} \mid Z_{j}^{\prime}, Z_{j}^{\prime \prime}\right)=p_{\mathcal{T}, q}\left(Y_{j 1}^{\prime} \mid Z_{j}^{\prime}\right) .
$$

and similarly for $Y_{j 1}^{\prime \prime}$. If $\mathbf{c}_{\tilde{\mathcal{T}}}\left(Z_{j}^{\prime} Y_{j 1}^{\prime}\right) \in \tilde{G}$ and $\mathbf{c}_{\tilde{\mathcal{T}}}\left(Z_{j}^{\prime \prime} Y_{j 1}^{\prime \prime}\right) \in \tilde{G}$, we have $Z_{j+1}^{\prime}$ and $Z_{j+1}^{\prime \prime}$. There is therefore no need for further samples $Y_{j 2}^{\prime}$ and $Y_{j 2}^{\prime \prime}$ onwards.

2) Sampling from $\mu\left(\left\{Y_{j i}^{\prime}, Y_{j i}^{\prime \prime}\right\}_{i \geq 2} \mid Z_{j}^{\prime} Y_{j 1}^{\prime}, Z_{j}^{\prime \prime} Y_{j 1}^{\prime \prime}\right)$ :

In case at least one of the following holds: $\mathbf{c}_{\tilde{\mathcal{T}}}\left(Z_{j}^{\prime} Y_{j 1}^{\prime}\right) \notin \tilde{G}$ or $\mathbf{c}_{\tilde{\mathcal{T}}}\left(Z_{j}^{\prime \prime} Y_{j 1}^{\prime \prime}\right) \notin \tilde{G}$, then the following subsection explains how to proceed. 
1) If $Y_{j 1}^{\prime}=Y_{j 1}^{\prime \prime}$ but only one of $\mathbf{c}_{\tilde{\mathcal{T}}}\left(Z_{j}^{\prime} Y_{j 1}^{\prime}\right) \in \tilde{G}$ and $\mathbf{c}_{\tilde{\mathcal{T}}}\left(Z_{j}^{\prime \prime} Y_{j 1}^{\prime \prime}\right) \in \tilde{G}$, then we have one of $Z_{j+1}^{\prime \prime}$ and $Z_{j+1}^{\prime \prime}$. To get the other, we continue (according to transitions defined by $p_{\mathcal{T}, q}$ ) only its corresponding chain till we get a good state.

2) If $Y_{j 1}^{\prime}=Y_{j 1}^{\prime \prime}, \mathbf{c}_{\tilde{\mathcal{T}}}\left(Z_{j}^{\prime} Y_{j 1}^{\prime}\right) \notin \tilde{G}$ and $\mathbf{c}_{\tilde{\mathcal{T}}}\left(Z_{j}^{\prime \prime} Y_{j 1}^{\prime \prime}\right) \notin \tilde{G}$, we need to continue both chains. We generate $Y_{j 2}^{\prime}, Y_{j 2}^{\prime \prime}$ as we did for the first samples - by generating a new random number $U_{j 2}$ uniform in $[0,1]$, and by coupling as in Fig. 7 (b) and the two distributions $q\left(\cdot \mid Z_{j}^{\prime} Y_{j 1}^{\prime}\right)$ and $q\left(\cdot \mid Z_{j}^{\prime \prime} \underline{Y}_{j 1}^{\prime \prime}\right)$. And continue in this fashion so long as the samples in the two chains remain equal but do not hit good contexts. This will be case that will be most important for us later on.

3) If $Y_{j l}^{\prime} \neq Y_{j l}^{\prime \prime}$ at any point and neither chain has seen a good state yet, we just run the chains independently from that point on for how long it takes each to hit a good aggregated state.

Once again we have

$$
\begin{aligned}
\mu\left(Y_{j(r+1)}^{\prime} \mid Z_{j}^{\prime} Y_{j 1}^{\prime}, \ldots, Y_{j r}^{\prime}, Z_{j}^{\prime \prime} Y_{j 1}^{\prime \prime}, \ldots, Y_{j r}^{\prime \prime}\right) & \\
& =p_{\mathcal{\tau}, q}\left(Y_{j(r+1)}^{\prime} \mid Z_{j}^{\prime} Y_{j 1}^{\prime}, \ldots, Y_{j r}^{\prime}\right),
\end{aligned}
$$

and similarly for $Y_{j(r+1)}^{\prime \prime}$.

\section{E. Some Observations on Coupling}

For any $r$, let $Z_{r}^{\prime} \sim Z_{r}^{\prime \prime}$ denote the following event that happens to be a subset of case where we do not need $Y_{r 2}^{\prime}$ and $Y_{r 2}^{\prime \prime}$ onwards,

$$
\left\{Y_{r 1}^{\prime}=Y_{r 1}^{\prime \prime} \text { and } \mathbf{c}_{\tilde{\mathcal{T}}}\left(Z_{r}^{\prime} Y_{r 1}^{\prime}\right) \in \tilde{G} \text { and } \mathbf{c}_{\tilde{\mathcal{T}}}\left(Z_{r}^{\prime \prime} Y_{r 1}^{\prime \prime}\right) \in \tilde{G}\right\} .
$$

Recall the definition of $\eta_{\tilde{G}}$ from [8].

Observation 2. $\mu\left(Z_{r}^{\prime} \sim Z_{r}^{\prime \prime} \mid Z_{r-1}^{\prime}, Z_{r-1}^{\prime \prime}\right) \geq \eta_{\tilde{G}}\left(1-\delta_{k_{n}}\right)$. Proof Combining (8) and (9), we have

$$
\begin{aligned}
\mu\left(Z_{r}^{\prime} \sim Z_{r}^{\prime \prime} \mid Z_{r-1}^{\prime}, Z_{r-1}^{\prime \prime}\right) & =\sum_{a \in \mathcal{A}} \min \left\{q\left(a \mid Z_{r-1}^{\prime}\right), q\left(a \mid Z_{r-1}^{\prime \prime}\right)\right\} \\
& \geq \eta_{\tilde{G}}\left(1-\delta_{k_{n}}\right) .
\end{aligned}
$$

Furthermore, if $Z_{i}^{\prime} \sim Z_{i}^{\prime \prime}$ for the $k_{n}$ consecutive samples, $j-k_{n}+1 \leq i \leq j$, then we have

$$
Z_{j}^{\prime} \stackrel{k_{n}}{\approx} Z_{j}^{\prime \prime}
$$

To proceed, once $Z_{j}^{\prime} \stackrel{k_{n}}{\approx} Z_{j}^{\prime \prime}$, we would like the two chains to coalesce tighter in every subsequent step, namely we want for all $1 \leq l \leq n, Z_{j+l}^{\prime} \stackrel{k_{n}+l}{\approx} Z_{j+l}^{\prime \prime}$. Starting from $Z_{j}^{\prime} \stackrel{k_{n}}{\approx} Z_{j}^{\prime \prime}$, we can have $Z_{j+1}^{\prime} \stackrel{k_{n}+1}{\approx} Z_{j+1}^{\prime \prime}$ if

1) $Z_{j+1}^{\prime} \sim Z_{j+1}^{\prime \prime}$, or

2) if the chains $\left\{Y_{j i}^{\prime}\right\}_{i \geq 1}$ and $\left\{Y_{j i}^{\prime \prime}\right\}_{i \geq 1}$ evolve through a sequence of $m>1$ steps before hitting a context in $\tilde{G}$ on the $m^{\prime}$ th step with $Y_{j l}^{\prime}=Y_{j l}^{\prime \prime}$ for each $l \leq m$.

Observation 3. Suppose $Z_{j}^{\prime} \stackrel{k_{n}}{\approx} Z_{j}^{\prime \prime}$. While sampling from $\mu$ for the samples $Z_{j+1}^{\prime}$ and $Z_{j+1}^{\prime \prime}$, suppose $Y_{j i}^{\prime}=Y_{j i}^{\prime \prime}$ for $i \geq 1$.
If $m$ is the first time the first chain hits a good context, namely $m$ is the smallest number such that

$$
\mathbf{c}_{\mathcal{A}^{k_{n}}}\left(Z_{j}^{\prime}\left\{Y_{j i}^{\prime}\right\}_{i=1}^{m}\right) \in \tilde{G},
$$

it follows that the second chain also hits a good context at the same time, namely

$$
\mathbf{c}_{\mathcal{A}^{k_{n}}}\left(Z_{j}^{\prime \prime}\left\{Y_{j i}^{\prime}\right\}_{i=1}^{m}\right)=\mathbf{c}_{\mathcal{A}^{k_{n}}}\left(Z_{j}^{\prime \prime}\left\{Y_{j i}^{\prime \prime}\right\}_{i=1}^{m}\right) \in \tilde{G} .
$$

Note that we may not be able to say the above if $Z_{j}^{\prime} \not \approx Z_{j}^{\prime \prime}$. Furthermore, now we also have $Z_{j+1}^{\prime} \stackrel{k_{n}+m}{\approx} Z_{j+1}^{\prime \prime}$.

Let us now bound how likely this sort of increasingly tighter merging is. Because of the way we have set up our coupling, the probability

$$
\begin{aligned}
\mu\left(Y_{j 1}^{\prime}=Y_{j 1}^{\prime \prime} \mid Z_{j}^{\prime} \stackrel{k_{n}}{\approx} Z_{j}^{\prime \prime}\right) & =\sum_{a \in \mathcal{A}} \min \left\{q\left(a \mid Z_{j}^{\prime}\right), q\left(a \mid Z_{j}^{\prime \prime}\right)\right\} \\
& \geq \sum_{a \in \mathcal{A}} \tilde{q}\left(a \mid \mathbf{c}_{\tilde{\mathcal{T}}}\left(Z_{j}^{\prime}\right)\right)\left(1-\delta_{k_{n}}\right) \\
& =1-\delta_{k_{n}},
\end{aligned}
$$

where $q$ and $\tilde{q}$ are the conditional probabilities associated with $p_{\mathcal{T}, q}$ and $p_{\tilde{\mathcal{T}}, \tilde{q}}$ respectively. Similarly

$$
\begin{aligned}
\mu\left(Y_{j(l+1)}^{\prime}=Y_{j(l+1)}^{\prime \prime} \mid Z_{j}^{\prime} \stackrel{k_{n}}{\approx} Z_{j}^{\prime \prime},\left\{Y_{j i}^{\prime}\right\}_{i=1}^{l}\right. & \left.=\left\{Y_{j i}^{\prime \prime}\right\}_{i=1}^{l}\right) \\
& \geq 1-\delta_{k_{n}+l} .
\end{aligned}
$$

It is important to note that the above statement holds whether

$$
\mathbf{c}_{\mathcal{A}^{k_{n}}}\left(Z_{j}^{\prime}\left\{Y_{j i}^{\prime}\right\}_{i=1}^{l}\right) \in \tilde{G} \text { or } \mathbf{c}_{\mathcal{A}^{k_{n}}}\left(Z_{j}^{\prime}\left\{Y_{j i}^{\prime}\right\}_{i=1}^{l}\right) \notin \tilde{G},
$$

and is simply a consequence of dependencies dying down. Therefore (no matter what $m$ is),

$$
\mu\left(Z_{j}^{\prime} \stackrel{k_{n}+1}{\approx} Z_{j}^{\prime \prime} \mid Z_{j-1}^{\prime} \stackrel{k_{n}}{\approx} Z_{j-1}^{\prime \prime}\right) \geq \prod_{l=k_{n}}^{\infty}\left(1-\delta_{l}\right) \stackrel{(i)}{\geq} 1-\Delta_{k_{n}},
$$

where the inequality in $(i)$ is by Proposition 2

The above equation, while accurate, is not the strongest we can say with essentially the same argument. We are now going to progressively make stronger statements with the same arguments. First, note that we obtain for all $\ell$, the event

$$
\left\{\exists \ell^{\prime} \leq \ell \text { s.t. } Z_{j+\ell^{\prime}}^{\prime} \stackrel{k_{n}+\ell}{\approx} Z_{j+\ell^{\prime}}^{\prime \prime}\right\},
$$

can happen by going through a sequence of tighter and tighter coalesced transitions of $p_{\mathcal{T}_{2 q}}$ (no matter in how many of those steps we saw contexts in $\tilde{G}$ ). Therefore,

$$
\begin{aligned}
\mu\left(\exists \ell^{\prime} \leq \ell \text { s.t. } Z_{j+\ell^{\prime}}^{\prime} \stackrel{k_{n}+\ell}{\approx} Z_{j+\ell^{\prime}}^{\prime \prime} \mid Z_{j}^{\prime} \stackrel{k_{n}}{\approx} Z_{j}^{\prime \prime}\right) & \geq \prod_{l=k_{n}}^{\infty}\left(1-\delta_{l}\right) \\
& \geq 1-\Delta_{k_{n}} .
\end{aligned}
$$

And we can easily strengthen the above to say for all $\ell$,

$$
\mu\left(Z_{j+\ell}^{\prime} \stackrel{k_{n}+\ell}{\approx} Z_{j+\ell}^{\prime \prime} \mid Z_{j}^{\prime} \stackrel{k_{n}}{\approx} Z_{j}^{\prime \prime}\right) \geq 1-\Delta_{k_{n}},
$$

for the same reason. Indeed, we can further strengthen the above statement to note: 
Observation 4. If $Z_{j}^{\prime} \stackrel{\ell}{\approx} Z_{j}^{\prime \prime}$ for any $\ell \geq k_{n}$, the chance of ever diverging,

$$
\mu\left(\exists l>0 \text { s.t. } Z_{j+l}^{\prime} \stackrel{\ell}{\not} Z_{j+l}^{\prime \prime} \mid Z_{j}^{\prime} \stackrel{\ell}{\approx} Z_{j}^{\prime \prime}\right) \leq \Delta_{\ell} .
$$

This motivates following definition of coalescence when dealing with finite length- $n$ samples.

Definition 7. We say the chains $\left\{Z_{i}^{\prime}\right\}_{i \geq 1}$ and $\left\{Z_{i}^{\prime \prime}\right\}_{i \geq 1}$ have coalesced if for any $j$,

$$
Z_{j}^{\prime} \stackrel{\max }{\left\{k_{n}, \ell_{n}\right\}} Z_{j}^{\prime \prime}
$$

where as in Definition 6. $\ell_{n}$ is the smallest number satisfying $\Delta_{\ell_{n}} \leq 1 / n$. Note that our definition of coalescence differs from regular literature which requires the equality in above definition.

\section{F. Deviation bounds on stationary probabilities}

Definition 8. Let

$$
\mathcal{B} \stackrel{\text { def }}{=} \mathcal{B}\left(k_{n}, \ell_{n}, \eta_{\tilde{G}}\right) \stackrel{\text { def }}{=} 1+\frac{4 \max \left\{\ell_{n}, k_{n}\right\}}{\eta_{\tilde{G}}^{k_{n}}\left(1-\Delta_{k_{n}}\right)},
$$

where $\ell_{n}$ is as in Definition 7 and $\eta_{\tilde{G}}$ is from 8 .

Remark The above quantity $\mathcal{B}$ will be a determining factor in how well we can estimate stationary probabilities. At this point, it may be useful to estimate the order of magnitude of the various terms. First, $k_{n}=\alpha_{n} \log n$ as mentioned before. The parameter $\eta_{\tilde{G}}<1$. The results below hold when $\eta_{\tilde{G}}$ is not too small, it should be a constant such that $\eta_{\tilde{G}}^{k_{n}}=\omega(1 / \sqrt{n})$. Since $k_{n}=\alpha_{n} \log n$, we typically can take $\alpha_{n}$ as large as a small constant in light of the above.

While the results below do not require it, it is helpful to keep the case $\tilde{n}=\mathcal{O}(n)$ in mind while interpreting the results below.

Note that if the dependencies die down exponentially, namely $d(i)=\gamma^{i}$ for some $0<\gamma<1$, then $\ell_{n}=$ $\left\lceil\log \left(n /(1-\gamma)^{2}\right) / \log 1 / \gamma\right\rceil$. If the dependencies die down polynomially, namely $d(i)=1 / i^{r}$ for some $r>2$, then $\ell_{n}=\left\lceil 2+\left(\frac{n}{(r-1)(r-2)}\right)^{\frac{1}{r-2}}\right\rceil$.

Lemma 5. Let $\left\{V_{m}\right\}_{m=0}^{\tilde{n}}$ be the Doob martingale described in Definition 6 For all $m \geq 1$,

$$
\left|V_{m}-V_{m-1}\right| \leq \mathcal{B}\left(k_{n}, \ell_{n}, \eta_{\tilde{G}}\right),
$$

where $\mathcal{B}\left(k_{n}, \ell_{n}, \eta_{\tilde{G}}\right)$ is as defined in Definition 8

Proof Recall that

$$
\begin{aligned}
& \left|V_{m}-V_{m-1}\right|=\left|\mathbb{E}\left[N_{\mathbf{w}} \mid Z_{0}, \ldots, Z_{m}\right]-\mathbb{E}\left[N_{\mathbf{w}} \mid Z_{0}, \ldots, Z_{m-1}\right]\right| \\
& \quad \leq \max _{Z_{m}^{\prime}, Z_{m}^{\prime \prime}}\left|\mathbb{E}\left[N_{\mathbf{w}} \mid Z_{0}, \ldots, Z_{m}^{\prime}\right]-\mathbb{E}\left[N_{\mathbf{w}} \mid Z_{0}, \ldots, Z_{m}^{\prime \prime}\right]\right| \\
& \quad \leq \sum_{j=m+1}^{n} \mu\left(Z_{j}^{\prime} \not ゙ Z_{j}^{\prime \prime}\right) .
\end{aligned}
$$

Let $\tau$ be the smallest number bigger than $m$ such that $Z_{\tau}^{\prime} \approx \ell_{n} Z_{\tau}^{\prime \prime}$. For any positive integer $t^{\prime}$, the probability $\tau>t^{\prime} \ell_{n}$ can be upper bounded by splitting the $t^{\prime} \ell_{n}$ samples of the two chains $\left\{Z_{j}^{\prime}\right\}_{j=m+1}^{m+t^{\prime} \ell_{n}}$ and $\left\{Z_{j}^{\prime \prime}\right\}_{j=m+1}^{m+t^{\prime} \ell_{n}}$ into blocks of length $\ell_{n}$. While the approach remains the same, we consider two calculations: (i) $k_{n} \leq \ell_{n}$ and (ii) $k_{n} \geq \ell_{n}$ below.

If $k_{n} \leq \ell_{n}$, the probability the two chains coalesce in any single block is, using Observation 3 for $k_{n}$ times and then Observation 4

$$
\begin{aligned}
& \geq \eta_{\tilde{G}}^{k_{n}}\left(1-\delta_{k_{n}}\right)^{k_{n}}\left(1-\Delta_{k_{n}}\right) \\
& \stackrel{(i)}{\geq} \eta_{\tilde{G}}^{k_{n}}\left(1-\frac{1}{k_{n}}\right)^{k_{n}}\left(1-\Delta_{k_{n}}\right) \\
& \geq \eta_{\tilde{G}}^{k_{n}}\left(1-\Delta_{k_{n}}\right) / 4,
\end{aligned}
$$

where $(i)$ is because $\delta_{k_{n}} \leq \frac{1}{k_{n}}$.

Thus,

$$
\mu\left(\tau>t^{\prime} \ell_{n}\right) \leq\left(1-\eta_{\tilde{G}}^{k_{n}}\left(1-\Delta_{k_{n}}\right) / 4\right)^{t^{\prime}} .
$$

Furthermore, $\mathbb{E} \tau-m$ can be bounded using the expected number of blocks before the chains merge in any single block, thus,

$$
\mathbb{E} \tau \leq m+\frac{4 \ell_{n}}{\eta_{\tilde{G}}^{k_{n}}\left(1-\Delta_{k_{n}}\right)} .
$$

Then for all $j \geq m+1$

$$
\begin{aligned}
\mu\left(Z_{j}^{\prime} \not \approx Z_{j}^{\prime \prime}\right) & =\mu\left(Z_{j}^{\prime \ell_{n}} \not \approx Z_{j}^{\prime \prime} \text { and } \tau<j\right)+\mu\left(Z_{j}^{\prime} \not \approx Z_{j}^{\prime \prime} \text { and } \tau \geq j\right) \\
& \stackrel{(i)}{\leq} \Delta_{\ell_{n}}+\mu\left(Z_{j}^{\prime} \not \approx Z_{j}^{\prime \prime} \text { and } \tau \geq j\right) \\
& \leq \frac{1}{n}+\mu(\tau \geq j),
\end{aligned}
$$

where inequality $(i)$ above follows because $Z_{\tau}^{\prime} \stackrel{\ell_{n}}{\approx} Z_{\tau}^{\prime \prime}$ by definition and from Observation 4 . Finally we upper bound (10),

$$
\begin{aligned}
\sum_{j=m+1}^{n} \mu\left(Z_{j}^{\prime} \not k_{n} Z_{j}^{\prime \prime}\right) & \leq \sum_{j=m+1}^{n} \mu\left(Z_{j}^{\prime} \not Z_{j}^{\prime \prime}\right) \\
& \leq n \cdot \frac{1}{n}+\sum_{j=m+1}^{n} \mu(\tau \geq j) \\
& \leq 1+\mathbb{E} \tau-m \\
& \leq 1+\frac{4 \ell_{n}}{\eta_{\tilde{G}}^{k_{n}}\left(1-\Delta_{k_{n}}\right)} .
\end{aligned}
$$

If $k_{n}>\ell_{n}$, we follow an identical line of argument with the exception that we divide the processes into blocks of $k_{n}$ samples, and $\tau$ as the first time the two processes satisfy $Z_{\tau}^{\prime} \stackrel{k_{n}}{\approx} Z_{\tau}^{\prime \prime}$. We then bound

$$
\begin{aligned}
& \mu\left(Z_{j}^{\prime} \not z_{n}^{k_{n}} Z_{j}^{\prime \prime}\right)=\mu\left(Z_{j}^{\prime k_{n}} \not Z_{j}^{\prime \prime} \text { and } \tau<j\right)+\mu\left(Z_{j}^{\prime k_{n}} \not Z_{j}^{\prime \prime} \text { and } \tau \geq j\right) \\
& \leq \frac{1}{n}+\mu(\tau \geq j)
\end{aligned}
$$

and finally obtain

$$
\begin{aligned}
\sum_{j=m+1}^{n} \mu\left(Z_{j}^{\prime k_{n}} \not ゙ Z_{j}^{\prime \prime}\right) & \leq n \cdot \frac{1}{n}+\sum_{j=m+1}^{n} \mu(\tau \geq j) \\
& \leq 1+\frac{4 k_{n}}{\eta_{\tilde{G}}^{k_{n}}\left(1-\Delta_{k_{n}}\right)} .
\end{aligned}
$$

Corollary 6. Let $\left\{V_{m}\right\}_{m=0}^{\tilde{n}}$ be the Doob martingale de- 
scribed in Definition 6 Then

$$
\left|V_{0}-\tilde{n} \frac{\pi(\mathbf{w})}{\pi(\tilde{G})}\right| \leq \mathcal{B}\left(k_{n}, \ell_{n}, \eta_{\tilde{G}}\right)
$$

where $\mathcal{B}\left(k_{n}, \ell_{n}, \eta_{\tilde{G}}\right)$ is as defined in Definition 8 ,

Proof We bound the value of $V_{0}=\mathbb{E}\left[N_{\mathbf{w}} \mid Z_{0}\right]$ by a coupling argument identical to Lemma 5. Suppose $\left\{Z_{m}^{\prime}\right\}$ and $\left\{Z_{m}^{\prime \prime}\right\}$ are coupled copies of the restriction of $p_{\mathcal{T}, q}$ to $\tilde{G}$, where $\left\{Z_{m}^{\prime}\right\}$ starts from state $Z_{0}$, while $\left\{Z_{m}^{\prime \prime}\right\}$ starts from a state chosen randomly according to the stationary distribution of $\left\{Z_{m}\right\}$. The same analysis holds and the Corollary follows using Property 2 in section XI

Theorem 7. Let $(\mathcal{T}, q(\mathcal{T}))$ be an unknown model in $\mathcal{M}_{d}$. If $\left\{Z_{m}\right\}_{m \geq 1}$ is aperiodic, then for any $t>0, Y_{-\infty}^{0}$ and $\mathbf{w} \in \tilde{G}$ we have

$$
p_{\mathcal{T}, q}\left(\left|N_{\mathbf{w}}-\tilde{n} \frac{\pi(\mathbf{w})}{\pi(\tilde{G})}\right| \geq t \mid Y_{-\infty}^{0}\right) \leq 2 \exp \left(-\frac{(t-\mathcal{B})^{2}}{2 \tilde{n} \mathcal{B}^{2}}\right),
$$

where $\mathcal{B}=\mathcal{B}\left(k_{n}, \ell_{n}, \eta_{\tilde{G}}\right)$ is as defined in 8 .

Proof Note that aperiodicity of the restriction $\left\{Z_{m}\right\}_{m \geq 1}$ of $p_{\mathcal{T}, q}$ to $\tilde{G}$ does not require an observation of $\left\{Z_{m}\right\}_{m \geq 1}$. We can check for this property using only $\tilde{G}$ as noted in Property 1

Theorem follows by Lemma 5, Corollary 6 and using Azuma's inequality.

Remark The theorem only has at most constant confidence if $t \leq \sqrt{\tilde{n}} \mathcal{B}$. Generally speaking,

$$
\mathcal{B} \approx \frac{\max \left\{k_{n}, \ell_{n}\right\}}{\eta_{\tilde{G}}^{k_{n}}\left(1-\Delta_{k_{n}}\right)} .
$$

For at least constant confidence, the deviation of the counts of w from $\pi(\mathbf{w}) / \pi(\tilde{G})$ is therefore

$$
\frac{t}{\tilde{n}} \approx \frac{\max \left\{k_{n}, \ell_{n}\right\}}{\sqrt{\tilde{n}} \eta_{\tilde{G}}^{k_{n}}\left(1-\Delta_{k_{n}}\right)} .
$$

For the exponential decay of dependencies, if $\tilde{n}=\mathcal{O}(n)$, we have both $k_{n}$ and $\ell_{n}$ of the order of $\log n$. Therefore, if $\eta_{\tilde{G}}$ is large enough that $\eta_{\tilde{G}}^{k_{n}} \geq n^{-\beta}$, namely

$$
\eta_{\tilde{G}} \geq \frac{\log n}{2^{\beta} k_{n}}
$$

for some constant $\beta<1 / 2$, the accuracy to which we can estimate the stationary probability ratio $\pi(\mathbf{w}) / \pi(\tilde{G})$ in Theorem 7 is

$$
\approx \frac{\log n}{n^{1 / 2-\beta}}
$$

We conclude with a couple of remarks and conjectures.

It is to be noted that for the concentration bounds on stationary probabilities to hold, we must have $\sum \delta_{i}<\infty$, something that was unnecessary for the transition probabilities. At this point, lacking a matching lower bound on deviation, we cannot say if this is an artifact of our arguments or if this is an interesting nuance that holds. However, we conjecture that estimating stationary probabilities is harder-that for the case $\sum \delta_{i}$ is not finite, we may be able to estimate only transition probabilities without ever estimating stationary probabilities.
Finally, to actually use Theorem 7, we further lower bound $\eta_{\tilde{G}}$ by estimates of aggregate transition probabilities derived from the data using Theorem 2 (or 4). With the effect that the model dependent right side in Theorem 7 is replaced by another upper bound-potentially worse, but entirely data dependent. The new data-only dependent upper bound holds with a reduced confidence obtained by a union bound on the confidences of Theorems 2 (or 4 ) and 7 .

Note also that the accuracy to which $\eta_{\tilde{G}}$ can be estimated is the same order of magnitude as the bound on the $\ell_{1}$ distance (between the naive and aggregated parameters) given in Theorems 2 (or 4). The accuracy in Theorems 2 (or 4) suffices, since we intend to use Theorem 7 when $\eta_{\tilde{G}}$ scales $\gg \frac{1}{\log n}$ (as mentioned before, we like $\eta_{\tilde{G}}$ to be $\Theta(1)$ ). While it is unclear if this scaling is a necessary for $\eta_{\tilde{G}}$, we believe this could be mildly improved on.

\section{Conclusions}

We have shown how to use data generated by potentially slow mixing Markov sources to identify those states for which naive approaches will estimate both parameters and functions related to stationary probabilities. To do so, we require that the underlying Markov source have dependencies that are not completely arbitrary, but die down eventually. In such cases, we show that even while the source may not have mixed (explored the state space properly), certain properties related to contexts $\mathbf{w}$, namely $\tilde{q}(. \mid \mathbf{w})$ or $\pi(\mathbf{w}) / \pi(\tilde{G})$ can be well estimated, if $|\mathbf{w}|$ grows as $\Theta(\log n)$. Surprisingly, we saw that it is quite possible that estimates related to contexts $\mathbf{w}$ may be good, even when estimates for suffixes of $\mathbf{w}$ fail-the reason being Theorem 2 depends not on the source mixing, but on the dependencies dying off. We also noted a couple of unanswered questions in our arguments-stationary probabilities seem to be harder to estimate, and we do not yet have a necessary condition on how large the parameter $\eta_{\tilde{G}}$ has to be for us to expect results.

This work also uncovers a lot of open problems. The above results are sufficient to say that some estimates are approximately accurate with high confidence. A natural, but perhaps difficult, question is whether we can give necessary conditions on how the data must look for a given estimate to be accurate. This work also forms a cog in the growing understanding of the information theoretic underpinnings involving estimation problems with memory. Finally, these results add to the understanding of model classes that only admit estimators converging pointwise over the class (namely at rates that could be arbitrarily slow depending on the underlying model), but are special in the sense that it is possible to say if the algorithm is doing well or not.

\section{APPENDIX A Proof of LEMMA 1}

For all $\mathbf{w} \in \tilde{\mathcal{T}}$, let $F(\mathbf{w})$ be the set of states $\mathbf{w}^{\prime} \in \tilde{\mathcal{T}}$ that reach $\mathbf{w}$ in one step, i.e.,

$$
F(\mathbf{w})=\left\{\mathbf{w}^{\prime} \in \tilde{\mathcal{T}}: \quad \exists a \in \mathcal{A} \quad \text { s.t. } \mathbf{w} \preceq \mathbf{w}^{\prime} a\right\} .
$$


Let $\tilde{Q}$ be the transition probability matrix formed by the states of $p_{\tilde{\mathcal{T}}, \tilde{q}}$. First notice that by definition, for all $\mathbf{w}, \mathbf{w}^{\prime} \in \tilde{\mathcal{T}}$,

$$
\tilde{Q}\left(\mathbf{w} \mid \mathbf{w}^{\prime}\right)= \begin{cases}\tilde{q}\left(a \mid \mathbf{w}^{\prime}\right) & \text { if } \mathbf{w}^{\prime} \in F(\mathbf{w}), \\ 0 & \text { if } \mathbf{w}^{\prime} \notin F(\mathbf{w})\end{cases}
$$

where $\tilde{q}$ is as in Definition 2. Since $p_{\mathcal{T}, q}$ is irreducible and aperiodic, $p_{\tilde{\tau} \tilde{q}}$ will also be irreducible and aperiodic. Thus, there is a unique stationary distribution $\tilde{\pi}$ corresponding to $p_{\tilde{\mathcal{T}, \tilde{q}}}$, i.e., unique solution for

$$
\tilde{\pi}(\mathbf{w})=\sum_{\mathbf{w}^{\prime} \in \tilde{\mathcal{T}}} \tilde{\pi}\left(\mathbf{w}^{\prime}\right) \tilde{Q}\left(\mathbf{w} \mid \mathbf{w}^{\prime}\right) \quad \forall \mathbf{w} \in \tilde{\mathcal{T}}
$$

We will consider a candidate solution of the form

$$
\tilde{\pi}(\mathbf{w})=\sum_{\mathbf{v} \in \mathcal{T}_{\mathbf{w}}} \pi(\mathbf{v})
$$

for every $\mathbf{w} \in \tilde{\mathcal{T}}$ and show that this candidate will satisfy 13 . Then, the claim will follow by uniqueness of the solution. To show this, note that for all $\mathrm{w} \in \tilde{\mathcal{T}}$,

$$
\begin{aligned}
\sum_{\mathbf{w}^{\prime} \in \tilde{\mathcal{T}}} \tilde{\pi}\left(\mathbf{w}^{\prime}\right) \tilde{Q}\left(\mathbf{w} \mid \mathbf{w}^{\prime}\right) & \\
& =\sum_{\mathbf{w}^{\prime} \in F(\mathbf{w})} \tilde{\pi}\left(\mathbf{w}^{\prime}\right) \tilde{q}\left(a \mid \mathbf{w}^{\prime}\right) \\
& =\sum_{\mathbf{w}^{\prime} \in F(\mathbf{w})}\left[\sum_{\mathbf{v} \in \mathcal{T}_{\mathbf{w}^{\prime}}} \pi(\mathbf{v})\right] \frac{\sum_{\mathbf{v} \in \mathcal{T}_{\mathbf{w}^{\prime}}} \pi(\mathbf{v}) q(a \mid \mathbf{v})}{\sum_{\mathbf{v}^{\prime} \in \mathcal{T}_{\mathbf{w}^{\prime}}} \pi\left(\mathbf{v}^{\prime}\right)} \\
& =\sum_{\mathbf{w}^{\prime} \in F(\mathbf{w})} \sum_{\mathbf{v} \in \mathcal{T}_{\mathbf{w}^{\prime}}} \pi(\mathbf{v}) q(a \mid \mathbf{v}) \\
& \stackrel{(i)}{=} \sum_{\mathbf{s} \in \mathcal{T}_{\mathbf{w}}} \pi(\mathbf{s}) \\
& \stackrel{(i i)}{=} \tilde{\pi}(\mathbf{w}),
\end{aligned}
$$

where $(i)$ holds because

$$
\bigcup_{\mathbf{w}^{\prime} \in F(\mathbf{w})} \mathcal{T}_{\mathbf{w}^{\prime}}=\mathcal{T}_{\mathbf{w}}
$$

and $(i i)$ follows from the definition of the proposed solution given in 14.

\section{APPENDIX B}

Proof of Proposition 1

Note that for all $\mathbf{w} \in \tilde{\mathcal{T}}, \mathcal{T}_{\mathbf{w}}=\{\mathbf{s} \in \mathcal{T}: \mathbf{w} \preceq \mathbf{s}\}$. Since $\tilde{\mathcal{T}} \preceq \mathcal{T}$, we have

$$
\begin{aligned}
\mathcal{H}_{\tilde{\mathcal{T}}} & =\sum_{\mathbf{w} \in \tilde{\mathcal{T}}} \tilde{\pi}(\mathbf{w}) \sum_{a \in \mathcal{A}} \tilde{q}(a \mid \mathbf{w}) \log \frac{1}{\tilde{q}(a \mid \mathbf{w})} \\
& \geq \sum_{\mathbf{w} \in \tilde{\mathcal{T}}} \tilde{\pi}(\mathbf{w}) \sum_{a \in \mathcal{A}} \sum_{\mathbf{v} \in \mathcal{T}_{\mathbf{w}}}\left[\frac{\pi(\mathbf{v})}{\sum_{\mathbf{v}^{\prime} \in \mathcal{T}_{\mathbf{w}}} \pi\left(\mathbf{v}^{\prime}\right)} q(a \mid \mathbf{v}) \log \frac{1}{q(a \mid \mathbf{v})}\right] \\
& \stackrel{(a)}{=} \sum_{\mathbf{w} \in \tilde{\mathcal{T}}} \sum_{a \in \mathcal{A}} \sum_{\mathbf{v} \in \mathcal{T}_{\mathbf{w}}} \pi(\mathbf{v}) q(a \mid \mathbf{v}) \log \frac{1}{q(a \mid \mathbf{v})} \\
& =\sum_{\mathbf{w} \in \tilde{\mathcal{T}}} \sum_{\mathbf{v} \in \mathcal{T}_{\mathbf{w}}} \pi(\mathbf{v}) \sum_{a \in \mathcal{A}} q(a \mid \mathbf{v}) \log \frac{1}{q(a \mid \mathbf{v})} \\
& =\sum_{\mathbf{s} \in \mathcal{T}} \pi(\mathbf{s}) \sum_{a \in \mathcal{A}} q(a \mid \mathbf{s}) \log \frac{1}{q(a \mid \mathbf{s})}=\mathcal{H}_{\mathcal{T}}
\end{aligned}
$$

where the first inequality follows because

$$
\tilde{q}(a \mid \mathbf{w})=\frac{\sum_{\mathbf{v} \in \mathcal{T}_{\mathbf{w}}} \pi(\mathbf{v}) q(a \mid \mathbf{v})}{\sum_{\mathbf{v}^{\prime} \in \mathcal{T}_{\mathbf{w}}} \pi\left(\mathbf{v}^{\prime}\right)},
$$

and because $g(x)=x \log \frac{1}{x}$ is concave for $x \in[0,1]$. The equality ( $a$ ) follows since $\tilde{\pi}(\mathbf{w})=\sum_{\mathbf{v} \in \mathcal{T}_{\mathbf{w}}} \pi(\mathbf{v})$.

\section{APPENDIX C}

\section{Proof OF Proposition 2}

Wolog, let $d(i)$ be decreasing, and consider a distribution $q$ over $\mathbb{N}$ such that

$$
\sum_{j \geq i} q(j)=d(i)
$$

Let $\left\{X_{n}\right\}_{n \geq 1}$ be a sequence of i.i.d. random variables distributed according to $q$ and let $E_{i}=P\left(X_{i} \geq i\right)$. Therefore, $E_{i}$ are independent with $\mathbb{P}\left(E_{i}\right)=d(i)$. Then,

$$
\mathbb{P}\left(\bigcup_{i \geq j} E_{i}\right)=1-\prod_{i \geq j}(1-d(i)) .
$$

Since

$$
\mathbb{P}\left(\bigcup_{i \geq j} E_{i}\right) \leq \sum_{i \geq j} \mathbb{P}\left(E_{i}\right)=\sum_{i \geq j} d(i)
$$

we have

$$
1-\sum_{i \geq j} d(i) \leq \prod_{i \geq j}(1-d(i)) .
$$

Since by assumption $0 \leq d(i) \leq 1$ for all $i \geq n_{0}$, the second inequality can easily be derived by the fact that

$$
\prod_{i \geq j}\left(1-d^{2}(i)\right) \leq 1
$$

\section{APPENDIX D \\ Proof of Proposition 3}

Let $\mathbf{w} \in \tilde{\mathcal{T}}$ and fix $a \in \mathcal{A}$. Note that for $\tilde{\mathcal{T}}=\mathcal{A}^{k_{n}}$, by assumption we have for all $b^{\prime}, b^{\prime \prime} \in \mathcal{A}$

$$
\left|\frac{q\left(a \mid b^{\prime} \mathbf{w}\right)}{q\left(a \mid b^{\prime \prime} \mathbf{w}\right)}-1\right| \leq d\left(k_{n}\right) .
$$


According to Lemma 1. $\tilde{q}(a \mid \mathbf{w})$ is weighted average of $q(a \mid b \mathbf{w}), b \in \mathcal{A}$. Hence,

$$
\min _{b \in \mathcal{A}} q(a \mid b \mathbf{w}) \leq \tilde{q}(a \mid \mathbf{w}) \leq \max _{b \in \mathcal{A}} q(a \mid b \mathbf{w}) .
$$

Combining (15) with $(16)$ and straightforward elementary algebra shows that $\forall b \in \mathcal{A}$

$$
\tilde{q}(a \mid \mathbf{w})\left(1-d\left(k_{n}\right)\right) \leq q(a \mid b \mathbf{w}) \leq\left(1+d\left(k_{n}\right)\right) \tilde{q}(a \mid \mathbf{w}) .
$$

Proceeding inductively, for all $\mathbf{s} \in \mathcal{T}_{\mathbf{w}}$ we have

$$
\begin{aligned}
\left(\prod_{i \geq k_{n}}(1-d(i))\right) \tilde{q}(a \mid \mathbf{w}) & \leq q(a \mid \mathbf{s}) \\
& \leq\left(\prod_{i \geq k_{n}}(1+d(i))\right) \tilde{q}(a \mid \mathbf{w}) .
\end{aligned}
$$

Now, Proposition 2 implies that

$$
\left(1-\sum_{i \geq k_{n}} d(i)\right) \max _{\mathbf{s} \in \mathcal{T}_{\mathbf{w}}} q(a \mid \mathbf{s}) \leq \tilde{q}(a \mid \mathbf{w}) \leq \frac{\min _{\mathbf{s} \in \mathcal{T}_{\mathbf{w}}} q(a \mid \mathbf{s})}{\left(1-\sum_{i \geq k_{n}} d(i)\right)} \text {. }
$$

\section{ACKNOWLEDGMENT}

This work was supported by NSF Grants CCF-1065632, CCF-1018984 and EECS-1029081. The authors thank Aleksander Kavčić and Rui Zhang at the University of Hawai' $i$, Mānoa for helpful discussions, and the anonymous reviewers for their comments and suggestions.

\section{REFERENCES}

[1] L. G. Valiant, "A theory of learnable," Communications of the ACM, vol. 27, no. 11, pp. 1134-1142, 1984.

[2] R. Motwani and P. Raghavan, Randomized Algorithms. Cambridge University Press, 1995.

[3] G. Grimmett and D. Stirzaker, Probability and Random Processes. Oxford University Press, 2001.

[4] T. Haveliwala and S. Kamvar, "The second eigenvalue of the Google matrix," Stanford University Technical Report, 2003.

[5] D. A. Levin, Y. Peres, and E. L. Wilmer, Markov Chains and Mixing Times. Americam Mathematical Society, 2009.

[6] D. J. Aldous, "Random walks on finite groups and rapidly mixing Markov chains," in Séminaire de Probabilités XVII - 1981/82, Springer Lecture Notes in Mathematics 986, 1983.

[7] B. Fittingoff, "Universal methods of coding for the case of unknown statistics," in Proceedings of the 5th Symposium on Information Theory. Moscow-Gorky, 1972, pp. 129-135.

[8] J. Shtarkov, "Coding of discrete sources with unknown statistics," in Topics in Information Theory (Coll. Math. Soc. J. Bolyai, no. 16), I. Csiszár and P. Elias, Eds. Amsterdam, The Netherlands: North Holland, 1977, pp. 559-574.

[9] R. Krichevsky and V. Trofimov, "The preformance of universal coding," IEEE Transactions on Information Theory, vol. 27, no. 2, pp. 199-207, March 1981.

[10] P. Laplace, Philosphical essays on probabilities, Translated by A. Dale from the 5th (1825) ed. Springer Verlag, New York, 1995.

[11] W. Gale and K. Church, "What is wrong with adding one?" in Corpus based research into language, N. Oostdijk and P. de Haan, Eds. Rodopi, Amsterdam, 1994, pp. 189-198.

[12] I. Good, "The population frequencies of species and the estimation of population parameters," Biometrika, vol. 40, no. 3/4, pp. 237-264, December 1953

[13] A. Orlitsky, N. Santhanam, and J. Zhang, "Always Good Turing: Asymptotically optimal probability estimation," in Proceedings of the 44th Annual Symposium on Foundations of Computer Sciece, October 2003.
[14] F. M. J. Willems, Y. M. Shtarkov, and T. J. Tjalkens, "The context-tree weighting method: basic properties." IEEE Transactions on Information Theory, vol. 41, no. 3, pp. 653-664, 1995.

[15] R. Krichevsky, Universal Compression and Retrieval. Kluwer Academic Publishers, 1993.

[16] B. Y. Ryabko, "Compression-based methods for nonparametric density estimation, on-line prediction, regression and classification for time series," in Information Theory Workshop, Porto, Portugal, 2008, pp. 271275.

[17] B. Ryabko, "Prediction of random sequences and universal coding," Problemy Peredachi Informatsii, vol. 24, no. 2, pp. 3-14, 1988.

[18] B. Y. Ryabko, "A fast adaptive coding algorithm," Problemy Peredachi Informatsii, vol. 26, no. 4, pp. 305-317, 1990.

[19] J. Rissanen, "Universal coding, information, prediction, and estimation," IEEE Transactions on Information Theory, vol. 30, no. 4, pp. 629-636, July 1984.

[20] M. Weinberger, J. Rissanen, and M. Feder, "A universal finite memory source," IEEE Transactions on Information Theory, vol. 41, no. 3, pp. 643-652, May 1995.

[21] T. J. Tjalkens, Y. Shtarkov, and F. Willems, "Sequential weighting algorithms for multi-alphabet sources," in 6th Joint Swedish-Russian International Workshop on Information Theory, August 1993, pp. 230 234.

[22] J. Kieffer, "A unified approach to weak universal source coding," IEEE Transactions on Information Theory, vol. 24, no. 6, pp. 674-682, November 1978.

[23] F. M. J. Willems, "The context-tree weighting method: Extensions," IEEE Transactions on Information Theory, vol. 44, pp. 792-798, 1998

[24] I. Csiszár and Z. Talata, "Context tree estimation for not necessarily finite memory processes, via BIC and MDL," IEEE Transactions on Information theory, vol. 52, no. 3, Mar 2006.

[25] A. Garivier, "Consistency of the unlimited BIC context tree estimator," IEEE Transactions on Information theory, vol. 52 , no. 10 , pp. 4630 4635, Sep 2006.

[26] A. Galves, V. Maume-Deschamps, and B. Schmitt, "Exponential inequalities for VLMC empirical trees," ESAIM: Probability and Statistics, vol. 12, pp. 219-229, Jan 2008.

[27] A. Garivier and F. Leonardi, "Context tree selection: A unifying view," Stochastic Processes and their Applications, vol. 121, no. 11, pp. 24882506, Nov 2011.

[28] J. Rissanen, "A universal data compression system," IEEE Transactions on Information theory, vol. 29, no. 5, pp. 656-664, Sep 1983.

[29] P. Bühlmann and A. Wyner, "Variable length Markov chains," Annals of Statistics, vol. 27, no. 2, pp. 480-583, 1999.

[30] G. Morvai and B. Weiss, "On sequential estimation and prediction for discrete time series," Stochastics and Dynamics, vol. 7, no. 4, pp. 417 437, 2007.

[31] I. Csiszár, "Large-scale typicality of Markov sample paths and consistency of MDL order estimators," IEEE Transactions on Information Theory, vol. 48, no. 6, pp. 1616-1628, Jun 2002.

[32] I. Csiszár and P. C. Shields, "The consistency of the BIC Markov order estimator," Annals of Statistics, vol. 28, pp. 1601-1619, 2000.

[33] I. Csiszár and Z. Talata, "On rate of convergence of statistical estimation of stationary ergodic processes," IEEE Transactions on Information Theory, vol. 56, no. 8, pp. 3637-3641, Aug 2010.

[34] A. Galves and F. Leonardi, "Exponential inequalities for empirical unbounded context trees," In and Out of Equilibrium 2, vol. 60, pp. 257-269, 2008.

[35] G. Morvai and B. Weiss, "Order estimation of Markov chains," IEEE Transactions on Information Theory, vol. 51, no. 4, pp. 1496-1497, 2005.

[36] R. van Handel, "On the minimal penalty for Markov order estimation," Probability theory and related fields, vol. 150, no. 3-4, pp. 709-738, 2011.

[37] N. Merhav, M. Gutman, and J. Ziv, "On the estimation of the order of a Markov chain and universal data compression," IEEE Transactions on Information Theory, vol. 35, no. 5, pp. 1014-1019, 1989.

[38] L. Finesso, L. Chuang-Chun, and P. Narayan, "The optimal error exponent for Markov order estimation," IEEE Transactions on Information Theory, vol. 42, no. 5, pp. 1488-1497, 1996.

[39] J. Ziv and A. Lempel, "Compression of individual sequences via variable-rate coding," IEEE Transactions on Information Theory, vol. 24, no. 5, pp. 530-536, Sep 1978.

[40] W. Feller, An Introduction to Probability Theory and Its Applications. John Wiley and Sons; 2nd Edition, 1957, vol. 1. 
[41] P. Ferrari and A. Galves, "Coupling and regeneration for stochastic processes," Notes for a minicourse presented in XIII Escuela Venezolana de Matematicas, 2000.

[42] V. Guruswami, "Rapidly mixing Markov chains: A comparison of techniques," Writeup at MIT Laboratory for Computer Science, 2000.

[43] T. Cover and J. Thomas, Elements of Information Theory. John Wiley and sons., 1991.

[44] D. Duarte, A. Galves, and N. L. Garcia, "Markov approximation and consistent estimation of unbounded probabilistic suffix trees," Bulletin of the Brazilian Mathematical Society, vol. 37, no. 4, pp. 581-592, 2006.

[45] P. Collet, A. Galves, and F. Leonardi, "Random perturbations of stochastic processes with unbounded variable length memory," Electronic Journal of Probability, vol. 13, pp. 1345-1361, 2008.

[46] B. Yu, "Rates of convergence for empirical processes of stationary mixing sequences," Annals of Probability, vol. 22, no. 1, pp. 94-116, 1994.

[47] J. R. Norris, Markov Chains. Cambridge Series in Statistical and Probabilistic Mathematics, 1998.

[48] S. Meyn and R. L. Tweedie, Markov Chains and Stochastic Stability. Cambridge University Press, 2009.

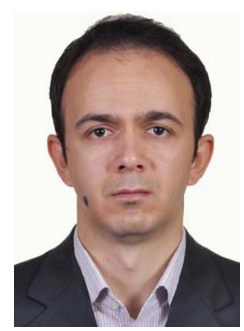

Meysam Asadi received his B.Sc. degree in Electrical Engineering from Razi University of Kermanshah, Iran, in 2004, followed by a M.Sc. degree at AmirKabir University, Tehran, Iran, in 2007, in Electrical Engineering and Computer Sciences. He is currently a Ph.D. candidate in the Department of Electrical Engineering at University of Hawai ‘i, Mānoa. His research interests cover estimating channels with memory, estimation in slow mixing Markov processes and detector design for storage systems.

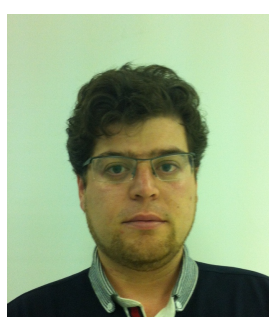

Ramezan Paravi Torghabeh was born in Mashhad, Iran, in 1982. He received Bachelor's degree from Ferdowsi University of Mashhad, Iran and Master's degree from K. N. Toosi University of Technology, Tehran, Iran both in Electrical Engineering in 2005 and 2008, respectively. He is currently a Ph.D. student in the Department of Electrical Engineering at University of Hawai ‘i, Mānoa.

His research interests include information theory, machine learning and probabilistic methods for high dimensional problems. He was recipient of Hawaiian Telcom Fellowship Fund in the area of telecommunications in 2011. He is a student member of the IEEE.

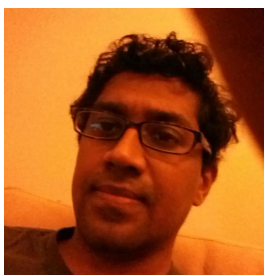

Narayana Santhanam is an Assistant Professor at the University of Hawaii since 2009. He obtained his B.Tech from the Indian Institute of Technology, Chennai (then Madras) in 2000; MS and PhD from the University of California, San Diego in 2003 and 2006 respectively. From 2007-2008, he held a postdoctoral position at the University of California, Berkeley.

His research interests lie in the intersection of information theory and statistics, with a focus on the undersampled/high dimensional regime and including applications to finance, biology, communication and estimation theory. $\mathrm{He}$ is the recipient of the 2006 Information Theory Best Paper award from the IEEE Information Theory Society along with A. Orlitsky and J. Zhang. He has co-organized several workshops on high dimensional statistics and "big data" problems over the last five years, and is a member of the NSF Center for Science of Information (CSoI), a NSF Science and Technology center. 